\title{
Initial-condition problem for a chiral Gross-Neveu system
}

\author{
P. L. Natti ${ }^{*}$ \\ Instituto de Física Teórica, Universidade Estadual Paulista, Rua Pamplona, 145-01405-900 São Paulo, São Paulo, Brazil
}

A. F. R. de Toledo Piza

Instituto de Física, Universidade de São Paulo, C.P. 66318, 05389-970 São Paulo, São Paulo, Brazil

(Received 12 December 1995; revised manuscript received 5 June 1996)

\begin{abstract}
A time-dependent projection technique is used to treat the initial-value problem for self-interacting fermionic fields. On the basis of the general dynamics of the fields, we derive formal equations of kinetic-type for the set of one-body dynamical variables. A nonperturbative mean-field expansion can be written for these equations. We treat this expansion in lowest order, which corresponds to the Gaussian mean-field approximation, for a uniform system described by the chiral Gross-Neveu Hamiltonian. Standard stationary features of the model, such as dynamical mass generation due to chiral symmetry breaking and a phenomenon analogous to dimensional transmutation, are reobtained in this context. The mean-field time evolution of nonequilibrium initial states is discussed. [S0556-2821(96)03322-X]
\end{abstract}

PACS number(s): 11.30.Rd, 11.10.Kk, 11.10.Lm

\section{INTRODUCTION}

Over the last two decades, interest in the initial-condition problem for field-theoretical models has been kindled and sustained by the needs of such apparently diverse areas as cosmology [1] and several branches of many-body physics, notably in connection with the analysis of transient phenomena in the collision of complex nuclear systems [2]. In these contexts one typically tries to obtain and solve equations describing the kinetic behavior of a particular, "relevant", subsystem or of a restricted set of "relevant" observables of a more comprehensive autonomous system. Such is the case, e.g., of the scalar driving field in the inflationary scenario of the early Universe and of one-body densities and certain correlation functions in heavy-ion collisions. In general, the relevant properties can be retrieved from appropriately constructed reduced density operators in the Schrödinger picture, which will evolve nonunitarily on account of correlation effects involving different subsystems $[3,4]$. The nonunitary effects will manifest themselves through the dynamical evolution of the eigenvalues of the reduced densities, so that particular subsystems will, in general, evolve in a nonisoentropic manner [3].

The overwhelming complexity of such a picture is considerably reduced whenever one is able to find physical grounds to motivate a mean-fieldlike approximation which consists in assuming isoentropic evolution of a relevant subsystem under effective, time-dependent Hamiltonian operators for each subsystem [4]. In this case the dynamics of the subsystem density matrix can be formulated in terms of a Liouville-von Neuman equation governed by an effective Hamiltonian and studied, e.g., from the point of view of the functional field-theoretical Schrödinger picture, as proposed by Jackiw [4]. Unfortunately, the resulting problem still involves, in general, nonlinear Hamiltonians, and cannot be

\footnotetext{
*Present address : Departamento de Física, Universidade Estadual de Londrina, Paraná, Brazil.
}

solved without further approximation. In the field-theoretical context, this has been implemented through the use of a Gaussian Ansatz for the subsystem density functional in the framework of a time-dependent variational principle supplying the appropriate dynamical information, notably for bosonic fields [5].

It is not difficult to see that this last approximation amounts to a second mean-field approximation, now at the microscopic level of the single-field, nonlinear, isoentropic effective dynamics. Actually, the Gaussian Ansatz, having the form of a exponential of a quadratic form in the field operators, implies that many-point correlation functions can be factored in terms of two-point functions. This is well known in the context of the derivation of the Hartree-Fock approximation to the nonrelativistic many-body problem [6]. This factorization has been used by Chang [7] to implement the Gaussian approximation for the $\lambda \phi^{4}$ theory. The dynamics of the reduced two-point density then itself becomes isoentropic, since irreducible higher-order correlation effects are neglected.

The focus of this work is a reevaluation of this second mean-field approximation, for fermionic fields, in terms of a time-dependent projection approach developed earlier for the nonrelativistic nuclear many-body dynamics by Nemes and de Toledo Piza [8]. This approach allows for the formulation of a mean-field expansion for the dynamics of the two-point correlation function from which one recovers the results of the Gaussian mean-field approximations in lowest order, i.e., this approach permits one to include and to evaluate higher dynamical correction effects to the simplest Gaussian meanfield approximation. Moreover, the expansion is energy conserving (for closed system) to all orders [9]. The resulting dynamical equations acquire the structure of kinetic equations which eliminate the isoentropic mean-field constraint describing the effective dynamics of a selected set of observables [8]. This approach was recently applied for the solution of the self-interacting $\lambda \phi^{4}$ theory in $1+1$ dimensions [10]. Lin and de Toledo Piza [10] find that the Gaussian meanfield approximation fails both qualitatively and quantitatively 
in the description of certain field variables. These failures are partially corrected by the collisional terms. Motivated by success obtained in description of time evolution of a offequilibrium uniform boson (scalar field) system beyond Gaussian mean-field approximation in quantum-field theoretical context, it becomes interesting to study the fermion case in this approach. As a first step towards this end we consider in this paper the implementation of the Gaussian approximation to a self-interacting system of fermions. This is done in a framework suitable for the subsequent inclusion of collisional (correlation) corrections along the lines developed in Ref. [10] for the self-interacting bosonic field.

We consider, for simplicity and definiteness, the case of an off-equilibrium, spatially uniform, many-fermion system described by chiral Gross-Neveu model [11]. This is an interesting nontrivial, renormalizable model for which many results are available in the literature so that it offers suitable testing ground for the proposed approach. On the basis of the general dynamics of the fields, we derive equations of kinetictype for the set of one-body variables in lowest order, which correspond to the Gaussian mean-field approximation. The detailed consideration of correlation corrections is deferred to future work.

An outline of the paper is as follows. In Sec. II we obtain the dynamical equations which describe the time evolution of a general uniform fermion system. These equations are the groundwork for the implementation of the time-dependent projection technique. This technique and the approximation scheme are described in Sec. III. In Sec. IV we implement in the quantum-field theoretical context the projection technique and obtain in Gaussian mean-field (isoentropic) approximation the equations which describe the effective dynamics of a off-equilibrium, spatially uniform, 1+1dimensional, self-interacting fermion system described by chiral Gross-Neveu model [11]. In Sec. V we use the static solution of these equations in order to renormalize the theory, leading to the well-known effective potential obtained by Gross and Neveu using the $1 / N$ expansion. In this same section, we show also that other static results which have been discussed in the literature, such as dynamical mass generation due to chiral symmetry breaking and a phenomenon analogous to dimensional transmutation, can be retrieved from this formulation in a mean-field approximation. Finally, in Sec. VI we obtain and discuss numerical solutions for nonequilibrium initial conditions. Section VII is devoted to a final discussion and conclusions. Some points of a more technical nature are discussed in Appendices.

\section{KINETICS OF A SELF-INTERACTING FERMIONIC FIELD}

In this section, we shall describe a formal treatment of the kinetics of a self-interacting quantum field. Although the procedure is quite general, we will adopt the specific context of a spatially uniform fermion system. We will illustrate all the relevant points of the approach and cut down inessential technical complications. Features of more general contexts are discussed in Ref. [12].

The idea of our approach is to focus on the time evolution of a set of simple observables. We argue that a large number of relevant physical observables are one-body operators.
Consequently, the time evolution of observables which involve field bilinear forms such as $\bar{\psi}(x) \psi(x), \psi(x) \psi(x), \ldots$ is desirable. These are the observables which are kept under direct control when one works variationally using a Gaussian functional Ansatz, and will, therefore, be referred to as Gaussian observables. In order to keep as close as possible to the formulation appropriate for the many-body problem, we work in fact with expressions which are bilinear in the creation and annihilation parts of the fields in momentum space with periodic boundary conditions in a spatial box of length $L$, defined in terms of an expansion mass parameter $m$. We begin by expanding the Dirac field operators $\psi(x)$ and $\bar{\psi}(x)$ in Heisenberg picture as

$\psi(x)=\sum_{\mathbf{k}}\left(\frac{m}{k_{0}}\right)^{1 / 2}\left[b_{\mathbf{k}, 1}(t) u_{1}(\mathbf{k}) \frac{e^{i \mathbf{k} \cdot \mathbf{x}}}{\sqrt{L}}+b_{\mathbf{k}, 2}^{\dagger}(t) u_{2}(\mathbf{k}) \frac{e^{-i \mathbf{k} \cdot \mathbf{x}}}{\sqrt{L}}\right]$,

$\bar{\psi}(x)=\sum_{\mathbf{k}}\left(\frac{m}{k_{0}}\right)^{1 / 2}\left[b_{\mathbf{k}, 1}^{\dagger}(t) \bar{u}_{1}(\mathbf{k}) \frac{e^{-i \mathbf{k} \cdot \mathbf{x}}}{\sqrt{L}}+b_{\mathbf{k}, 2}(t) \bar{u}_{2}(\mathbf{k}) \frac{e^{i \mathbf{k} \cdot \mathbf{x}}}{\sqrt{L}}\right]$,

where $b_{\mathbf{k}, 1}^{\dagger}$ and $b_{\mathbf{k}, 1}\left[b_{\mathbf{k}, 2}^{\dagger}\right.$ and $\left.b_{\mathbf{k}, 2}\right]$ are fermion creation and annihilation operators associated with positive (negative)energy solutions $u_{1}(\mathbf{k})\left[u_{2}(\mathbf{k})\right]$ of Dirac's equation. Canonical quantization demands that the creation and annihilation operators satisfy the standard anticommutation relations at equal times

$$
\begin{aligned}
& \left\{b_{\mathbf{k}, \lambda}^{\dagger}(t), b_{\mathbf{k}^{\prime}, \lambda^{\prime}}\left(t^{\prime}\right)\right\}_{t=t^{\prime}}=\delta_{\mathbf{k}, \mathbf{k}^{\prime}} \delta_{\lambda, \lambda^{\prime}} \quad \text { for } \lambda, \lambda^{\prime}=1,2, \\
& \left\{b_{\mathbf{k}, \lambda}^{\dagger}(t), b_{\mathbf{k}^{\prime}, \lambda^{\prime}}^{\dagger}\left(t^{\prime}\right)\right\}_{t=t^{\prime}}=\left\{b_{\mathbf{k}, \lambda}(t), b_{\mathbf{k}^{\prime}, \lambda^{\prime}}\left(t^{\prime}\right)\right\}_{t=t^{\prime}}=0 .
\end{aligned}
$$

In Eq. (1) $\mathbf{x}$ denotes the spatial coordinate only and we use the notation

$$
\left(k_{0}\right)^{2}=(\mathbf{k})^{2}+m^{2} \quad \text { and } k x=k_{0} t-\mathbf{k} \cdot \mathbf{x} .
$$

In general, the state of the system is given in terms of a many-body density operator $\mathcal{F}$ in the Heisenberg picture, a time-independent, non-negative, Hermitian operator with unit trace. At this point, however, we specialize the analysis to the case of spatially uniform systems. These systems exhibit translational invariance (homogeneity) and rotational invariance (isotropy). In the case of $1+1$ dimensions this reduces to invariance under translations and under reflection. The possible nonvanishing mean values of bilinear forms of field operators are

$$
\begin{array}{cc}
R_{\mathbf{k}, \lambda^{\prime} ; \mathbf{k}, \lambda}(t)=\operatorname{Tr}\left\{\left[b_{\mathbf{k}, \lambda^{\prime}}^{\dagger}(t) b_{\mathbf{k}, \lambda}(t)\right] \mathcal{F}\right\} & \text { for } \lambda, \lambda^{\prime}=1,2, \\
\Pi_{\mathbf{k}, \lambda^{\prime} ; \mathbf{k}, \lambda}(t)=\operatorname{Tr}\left\{\left[b_{-\mathbf{k}, \lambda^{\prime}}(t) b_{\mathbf{k}, \lambda}(t)\right] \mathcal{F}\right\} & \text { for } \lambda, \lambda^{\prime}=1,2
\end{array}
$$

The Hermitian matrix $R$ and the antisymmetric matrix $\Pi$ are the one-fermion density and pairing density, respectively. Using these objects we can construct the extended one-body density [13] 


$$
\begin{aligned}
\mathcal{R}_{\mathbf{k}}(t) & =\left[\begin{array}{cc}
R_{\mathbf{k}}(t) & \Pi_{\mathbf{k}}(t) \\
-\Pi_{\mathbf{k}}^{*}(t) & I_{2}-R_{\mathbf{k}}^{*}(t)
\end{array}\right] \\
& =\left[\begin{array}{cc}
\left\langle b_{\mathbf{k}, \lambda^{\prime}}^{\dagger}(t) b_{\mathbf{k}, \lambda}(t)\right\rangle & \left\langle b_{-\mathbf{k}, \lambda^{\prime}}(t) b_{\mathbf{k}, \lambda}(t)\right\rangle \\
\left\langle b_{-\mathbf{k}, \lambda^{\prime}}^{\dagger}(t) b_{\mathbf{k}, \lambda}^{\dagger}(t)\right\rangle & \left\langle b_{\mathbf{k}, \lambda^{\prime}}(t) b_{\mathbf{k}, \lambda}^{\dagger}(t)\right\rangle
\end{array}\right] .
\end{aligned}
$$

This object summarizes all information on the Gaussian observables and provides an adequate starting point for our kinetic treatment.

The first step is standard and consists in reducing the extended one-body density to diagonal form. This can be achieved by subjecting the creation and annihilation operators to a canonical transformation of the Bogolyubov-type with coefficients defined by the eigenvalue problem

$$
\mathcal{X}_{\mathbf{k}}^{\dagger}(t) \mathcal{R}_{\mathbf{k}}(t) \mathcal{X}_{\mathbf{k}}(t)=Q_{\mathbf{k}}(t),
$$

where the unitary matrix $\mathcal{X}_{\mathbf{k}}(t)$ which diagonalizes $\mathcal{R}_{\mathbf{k}}(t)$ has the structure

$$
\mathcal{X}_{\mathbf{k}}=\left[\begin{array}{cc}
X_{\mathbf{k}}^{*} & Y_{\mathbf{k}}^{*} \\
Y_{\mathbf{k}} & X_{\mathbf{k}}
\end{array}\right], \quad \mathcal{X}_{\mathbf{k}}^{\dagger}=\left[\begin{array}{cc}
X_{\mathbf{k}}^{T} & Y_{\mathbf{k}}^{\dagger} \\
Y_{\mathbf{k}}^{T} & X_{\mathbf{k}}^{\dagger}
\end{array}\right],
$$

and $Q_{\mathbf{k}}(t)$ is a diagonal matrix which can be written as

$$
\begin{aligned}
Q_{\mathbf{k}}(t) & =\left[\begin{array}{cc}
\nu_{\mathbf{k}}(t) & 0 \\
0 & I_{2}-\nu_{\mathbf{k}}(t)
\end{array}\right] \\
& =\left[\begin{array}{cc}
\left\langle\beta_{\mathbf{k}, \lambda^{\prime}}^{\dagger}(t) \beta_{\mathbf{k}, \lambda}(t)\right\rangle & \left\langle\beta_{-\mathbf{k}, \lambda^{\prime}}(t) \beta_{\mathbf{k}, \lambda}(t)\right\rangle \\
\left\langle\beta_{-\mathbf{k}, \lambda^{\prime}}^{\dagger}(t) \beta_{\mathbf{k}, \lambda}^{\dagger}(t)\right\rangle & \left\langle\beta_{\mathbf{k}, \lambda^{\prime}}(t) \beta_{\mathbf{k}, \lambda}^{\dagger}(t)\right\rangle
\end{array}\right] .
\end{aligned}
$$

The unitary conditions for $\mathcal{X}_{\mathbf{k}}(t)$ can be interpreted as orthogonality and completeness relations for a set of natural orbitals which diagonalize the extended one-body density. They read

$$
\mathcal{X}_{\mathbf{k}}^{\dagger} \mathcal{X}_{\mathbf{k}}=I_{4} \quad \text { and } \quad \mathcal{X}_{\mathbf{k}} \mathcal{X}_{\mathbf{k}}^{\dagger}=I_{4}
$$

or, more explicitly, in terms of the submatrices $X_{\mathbf{k}}$ and $Y_{\mathbf{k}}$ [see Eq. (6)],

$$
\begin{array}{ll}
Y_{\mathbf{k}} Y_{\mathbf{k}}^{\dagger}+X_{\mathbf{k}} X_{\mathbf{k}}^{\dagger}=\mathbf{I}_{2}, & Y_{\mathbf{k}} X_{\mathbf{k}}^{T}+X_{\mathbf{k}} Y_{\mathbf{k}}^{T}=\mathbf{0}_{2}, \\
Y_{\mathbf{k}}^{\dagger} Y_{\mathbf{k}}+X_{\mathbf{k}}^{T} X_{\mathbf{k}}^{*}=\mathbf{I}_{2}, & Y_{\mathbf{k}}^{T} X_{\mathbf{k}}^{*}+X_{\mathbf{k}}^{\dagger} Y_{\mathbf{k}}=\mathbf{0}_{2} .
\end{array}
$$

The elements of the diagonal submatrix $\nu_{\mathbf{k}}(t)$ can, on the other hand, be interpreted as quasifermion occupation numbers for the paired natural orbitals. Because of the assumed symmetry one must have

$$
\nu_{\mathbf{k}, \lambda}(t)=\nu_{-\mathbf{k}, \lambda}(t) .
$$

Finally, using Eq. (6), we can relate the fermion operators $b_{\mathbf{k}, \lambda}^{\dagger}(t)$ and $b_{\mathbf{k}, \lambda}(t)$ to the new quasifermion operators $\beta_{\mathbf{k}, \lambda}^{\dagger}(t)$ and $\beta_{\mathbf{k}, \lambda}(t)$ for $\lambda=1,2$ as

$$
\left[\begin{array}{c}
b_{\mathbf{k}, 1} \\
b_{\mathbf{k}, 2} \\
b_{-\mathbf{k}, 1}^{\dagger} \\
b_{-\mathbf{k}, 2}^{\dagger}
\end{array}\right]=\left[\begin{array}{cccc}
X_{11} & X_{21} & Y_{11}^{*} & Y_{21}^{*} \\
X_{12} & X_{22} & Y_{12}^{*} & Y_{22}^{*} \\
Y_{11} & Y_{21} & X_{11}^{*} & X_{21}^{*} \\
Y_{12} & Y_{22} & X_{12}^{*} & X_{22}^{*}
\end{array}\right]\left[\begin{array}{c}
\beta_{\mathbf{k}, 1} \\
\beta_{\mathbf{k}, 2} \\
\beta_{-\mathbf{k}, 1}^{\dagger} \\
\beta_{-\mathbf{k}, 2}^{\dagger}
\end{array}\right] .
$$

With the help of Eq. (11) it is then an easy task to express $\bar{\psi}(x)$ and $\psi(x)$, Eq. (1), in term of $\beta_{\mathbf{k}, \lambda}^{\dagger}(t)$ and $\beta_{\mathbf{k}, \lambda}(t)$ for $\lambda=1,2$. In doing so, one finds that the plane waves of $\bar{\psi}(x)$ and $\psi(x)$ are modified by a complex, momentumdependent redefinition of $m$ involving the Bogolyubov parameters. The complex character of these parameters is actually crucial in dynamical situations, where the imaginary parts will allow for the description of time-odd (i.e., velocitylike) properties.

What we have achieved so far amounts to obtaining an expansion of the fields $\bar{\psi}(x)$ and $\psi(x)$ such that the mean values in $\mathcal{F}$ of Gaussian observables are parametrized in terms of the natural orbitals $X_{\lambda^{\prime}, \lambda}(\mathbf{k})$ and $Y_{\lambda^{\prime}, \lambda}(\mathbf{k})$ and of the occupation numbers $\nu_{\mathbf{k}, \lambda}(t)=\operatorname{Tr}\left(\beta_{\mathbf{k}, \lambda}^{\dagger} \beta_{\mathbf{k}, \lambda} \mathcal{F}\right)$ for $\lambda=1,2$. In general, all these quantities are time dependent under the Heisenberg dynamics of the field operators, and we now proceed to obtain the corresponding equations of motion. Taking the time derivative of Eq. (5) and using the unitarity condition (8), we get

$$
\mathcal{X}_{\mathbf{k}}^{\dagger} \dot{\mathcal{R}}_{\mathbf{k}} \mathcal{X}_{\mathbf{k}}=\dot{Q}_{\mathbf{k}}-\dot{\mathcal{X}}_{\mathbf{k}}^{\dagger} \mathcal{X}_{\mathbf{k}} Q_{\mathbf{k}}-Q_{\mathbf{k}} \mathcal{X}_{\mathbf{k}}^{\dagger} \dot{\mathcal{X}}_{\mathbf{k}}
$$

We next evaluate the left-hand side of this equation using the Heisenberg equation of motion to obtain

$$
\begin{aligned}
& i \mathcal{X}_{\mathbf{k}}^{\dagger} \mathcal{\mathcal { R }}_{\mathbf{k}} \mathcal{X}_{\mathbf{k}} \\
& \quad=\left[\begin{array}{cc}
\operatorname{Tr}\left(\left[\beta_{\mathbf{k}, \lambda^{\prime}}^{\dagger} \beta_{\mathbf{k}, \lambda}, H\right] \mathcal{F}\right) & \operatorname{Tr}\left(\left[\beta_{-\mathbf{k}, \lambda^{\prime}} \beta_{\mathbf{k}, \lambda}, H\right] \mathcal{F}\right) \\
\operatorname{Tr}\left(\left[\beta_{-\mathbf{k}, \lambda}^{\dagger}, \beta_{\mathbf{k}, \lambda}^{\dagger}, H\right] \mathcal{F}\right) & \operatorname{Tr}\left(\left[\beta_{\mathbf{k}, \lambda^{\prime}} \beta_{\mathbf{k}, \lambda}^{\dagger}, H\right] \mathcal{F}\right)
\end{array}\right] .
\end{aligned}
$$

The right-hand side of Eq. (12) can also be evaluated explicitly using Eqs. (6) and (7):

$$
\begin{aligned}
& i\left(\dot{Q}_{\mathbf{k}}-\dot{\mathcal{X}}_{\mathbf{k}}^{\dagger} \mathcal{X}_{\mathbf{k}} Q_{\mathbf{k}}-Q_{\mathbf{k}} \mathcal{X}_{\mathbf{k}}^{\dagger} \dot{\mathcal{X}}_{\mathbf{k}}\right) \\
& \quad=\left[\begin{array}{cc}
i \dot{\nu}_{\mathbf{k}}+\left[\nu_{\mathbf{k}}, h_{\mathbf{k}}^{*}\right]_{-} & -g_{\mathbf{k}}^{*}+\left\{\nu_{\mathbf{k}}, g_{\mathbf{k}}^{*}\right\}_{+} \\
-g_{\mathbf{k}}+\left\{\nu_{\mathbf{k}}, g_{\mathbf{k}}\right\}_{+} & -i \dot{\nu}_{\mathbf{k}}+\left[\nu_{\mathbf{k}}, h_{\mathbf{k}}\right]_{-}
\end{array}\right],
\end{aligned}
$$

where the matrices $h_{\mathbf{k}}$ and $g_{\mathbf{k}}$ are given in terms of $X_{\mathbf{k}}$ and $Y_{\mathbf{k}}$ as

$$
\begin{aligned}
& h_{\mathbf{k}}=-i\left(\dot{Y}_{\mathbf{k}}^{T} Y_{\mathbf{k}}^{*}+\dot{X}_{\mathbf{k}}^{\dagger} X_{\mathbf{k}}\right), \\
& g_{\mathbf{k}}=-i\left(\dot{Y}_{\mathbf{k}}^{T} X_{\mathbf{k}}^{*}+\dot{X}_{\mathbf{k}}^{\dagger} Y_{\mathbf{k}}\right) .
\end{aligned}
$$

From Eqs. (13) and (14) we obtain dynamical equations which describe the time evolution of our uniform fermion system. They read 


$$
\begin{gathered}
i \dot{\nu}_{\mathbf{k}}+\left[\nu_{\mathbf{k}}, h_{\mathbf{k}}^{*}\right]_{-}=\operatorname{Tr}\left(\left[\beta_{\mathbf{k}, \lambda^{\prime}}^{\dagger} \beta_{\mathbf{k}, \lambda}, H\right] \mathcal{F}\right), \\
-g_{\mathbf{k}}^{*}+\left\{\nu_{\mathbf{k}}, g_{\mathbf{k}}^{*}\right\}_{+}=\operatorname{Tr}\left(\left[\beta_{-\mathbf{k}, \lambda^{\prime}} \beta_{\mathbf{k}, \lambda}, H\right] \mathcal{F}\right) .
\end{gathered}
$$

Equations (16), together with the unitarity conditions (9), determine the time rate of change of the Gaussian observables in terms of expectation values of appropriate commutators. They are, however, clearly not closed equations when the Hamiltonian $H$ involves self-interacting fields. In this case, in fact, the time derivatives of the Gaussian observables are given in terms of traces which are not expressible in terms of the Gaussian observables themselves, since they will involve also many-fermion densities. This situation can be dealt with in terms of the projection technique reviewed in the next section.

\section{PROJECTION TECHNIQUE AND APPROXIMATION SCHEME}

In this section we introduce the time-dependent projection technique [8] which permits one to obtain closed approximations to the equations of motion (16). It has been developed earlier in the context of nonrelativistic nuclear many-body dynamics and was recently applied in the quantum-field theoretical context to the self-interacting $\lambda \phi^{4}$ theory in $1+1$ dimensions [10]. It allows for the formulation of a meanfield expansion for the dynamics of the two-point correlation function from which one recovers the results of the Gaussian mean-field approximations in lowest order. If carried to higher orders it allows for the inclusion and evaluation of higher dynamical correlation corrections to the simplest mean-field approximation.

In the specific context of the equations of motion (16) we begin by decomposing the full density $\mathcal{F}$ as

$$
\mathcal{F}=\mathcal{F}_{0}(t)+\mathcal{F}^{\prime}(t)
$$

where $\mathcal{F}_{0}(t)$ is a Gaussian Ansatz which achieves a HartreeFock factorization of traces involving more than two field operators. The Gaussian density $\mathcal{F}_{0}(t)$ is chosen as having the form of a exponential of a bilinear, Hermitian expression in the fields normalized to unit trace [6]. In the momentum basis, it reads

$$
\mathcal{F}_{0}=\frac{\exp \left[\Sigma_{\left(\mathbf{k}_{1}, \mathbf{k}_{2}\right)} A_{\mathbf{k}_{1}, \mathbf{k}_{2}} b_{\mathbf{k}_{1}}^{\dagger} b_{\mathbf{k}_{2}}+B_{\mathbf{k}_{1}, \mathbf{k}_{2}} b_{\mathbf{k}_{1}}^{\dagger} b_{\mathbf{k}_{2}}^{\dagger}+C_{\mathbf{k}_{1}, \mathbf{k}_{2}} b_{\mathbf{k}_{1}} b_{\mathbf{k}_{2}}\right]}{\operatorname{Tr}\left\{\exp \left[\Sigma_{\left(\mathbf{k}_{1}, \mathbf{k}_{2}\right)} A_{\mathbf{k}_{1}, \mathbf{k}_{2}} b_{\mathbf{k}_{1}}^{\dagger} b_{\mathbf{k}_{2}}+B_{\mathbf{k}_{1}, \mathbf{k}_{2}} b_{\mathbf{k}_{1}}^{\dagger} b_{\mathbf{k}_{2}}^{\dagger}+C_{\mathbf{k}_{1}, \mathbf{k}_{2}} b_{\mathbf{k}_{1}} b_{\mathbf{k}_{2}}\right]\right\}} .
$$

The parameters in Eq. (18) are fixed by requiring that mean values in $\mathcal{F}_{0}$ of expressions that are bilinear in the fields reproduce the corresponding $\mathcal{F}$ averages [see Eqs. (20) below]. $\mathcal{F}_{0}$ is a time-dependent object, which acquires a particularly simple form when expressed in terms of the Bogolyubov quasifermion operators

$$
\mathcal{F}_{0}(t)=\prod_{\mathbf{k}, \lambda}\left[\nu_{\mathbf{k}, \lambda} \beta_{\mathbf{k}, \lambda}^{\dagger}(t) \beta_{\mathbf{k}, \lambda}(t)+\left(1-\nu_{\mathbf{k}, \lambda}\right) \beta_{\mathbf{k}, \lambda}(t) \beta_{\mathbf{k}, \lambda}^{\dagger}(t)\right] .
$$

This is clearly a unit trace object which, in addition, satisfies

$$
\begin{gathered}
\operatorname{Tr}\left(\beta_{a} \mathcal{F}_{0}\right)=\operatorname{Tr}\left(\beta_{a} \mathcal{F}\right)=\operatorname{Tr}\left(\beta_{a}^{\dagger} \mathcal{F}_{0}\right)=\operatorname{Tr}\left(\beta_{a}^{\dagger} \mathcal{F}\right)=0, \\
\operatorname{Tr}\left(\beta_{a} \beta_{b} \mathcal{F}_{0}\right)=\operatorname{Tr}\left(\beta_{a} \beta_{b} \mathcal{F}\right)=0, \\
\operatorname{Tr}\left(\beta_{a}^{\dagger} \beta_{b}^{\dagger} \mathcal{F}_{0}\right)=\operatorname{Tr}\left(\beta_{a}^{\dagger} \beta_{b}^{\dagger} \mathcal{F}\right)=0, \\
\operatorname{Tr}\left(\beta_{a}^{\dagger} \beta_{b} \mathcal{F}_{0}\right)=\operatorname{Tr}\left(\beta_{a}^{\dagger} \beta_{b} \mathcal{F}\right)=\nu_{a} \delta_{a, b}
\end{gathered}
$$

and

$$
\operatorname{Tr}\left(\beta_{a} \beta_{b}^{\dagger} \mathcal{F}_{0}\right)=\operatorname{Tr}\left(\beta_{a} \beta_{b}^{\dagger} \mathcal{F}\right)=\left(1-\nu_{a}\right) \delta_{a, b} .
$$

Correspondingly, the "remainder', density $\mathcal{F}^{\prime}(t)$, defined by Eq. (17), is a traceless, pure correlation density. As already remarked, a crucial point to observe is that $\mathcal{F}_{0}(t)$ can be written as a time-dependent projection of $\mathcal{F}$ : i.e.,

$$
\mathcal{F}_{0}(t)=\mathcal{P}(t) \mathcal{F} \quad \text { with } \mathcal{P}(t) \mathcal{P}(t)=\mathcal{P}(t) .
$$

In order to completely define this projector we require further that it satisfies

$$
i \dot{\mathcal{F}}_{0}(t)=[\mathcal{P}(t), \mathcal{L}] \mathcal{F}=\left[\mathcal{F}_{0}(t), H\right]+\mathcal{P}(t)[H, \mathcal{F}],
$$

where $\mathcal{L}$ is the Liouvillian defined as

$$
\mathcal{L} \cdot=[H, \cdot],
$$

$H$ being the Hamiltonian of the field. Equation (22) is just the Heisenberg picture counterpart of the condition $\dot{\mathcal{P}}(t) \mathcal{F}=0$ which has been used to define $\mathcal{P}(t)$ in the Schrödinger picture [9]. It is possible to show that conditions (21) and (22) make $\mathcal{P}(t)$ unique and we obtain an explicit form for this object in terms of the quasifermion operators and of the natural orbital occupations $[3,8-10]$.

The existence of the projector $\mathcal{P}(t)$ allows one to obtain an equation relating the correlation part $\mathcal{F}^{\prime}(t)$ to the Gaussian part $\mathcal{F}_{0}(t)$ of the full density. This can be immediately obtained from Eqs. (17), (21), and (22) and reads

$$
\left[i \partial_{t}+\mathcal{P}(t) \mathcal{L}\right] \mathcal{F}^{\prime}(t)=[\mathcal{I}-\mathcal{P}(t)] \mathcal{L F}_{0}(t)
$$

This equation has the formal solution

$$
\mathcal{F}^{\prime}(t)=\mathcal{G}(t, 0) \mathcal{F}^{\prime}(0)-i \int_{0}^{t} d t^{\prime} \mathcal{G}\left(t, t^{\prime}\right)\left[\mathcal{I}-\mathcal{P}\left(t^{\prime}\right)\right] \mathcal{L F}_{0}\left(t^{\prime}\right)
$$


where the first term accounts for initial correlations possibly contained in $\mathcal{F}$. The object $\mathcal{G}\left(t, t^{\prime}\right)$ is the time-ordered Green's function

$$
\mathcal{G}\left(t, t^{\prime}\right)=T\left(\exp \left[i \int_{t^{\prime}}^{t} d \tau \mathcal{P}(\tau) \mathcal{L}\right]\right) .
$$

We see thus that $\mathcal{F}^{\prime}(t)$, and, therefore, also $\mathcal{F}$ [see Eq. (17)], can be formally expressed in terms of $\mathcal{F}_{0}\left(t^{\prime}\right)$ (for $t^{\prime} \leqslant t$ ) and of initial correlations $\mathcal{F}^{\prime}(0)$. This allows us to express also the dynamical equations (16) as functionals of $\mathcal{F}_{0}\left(t^{\prime}\right)$ and of the initial correlations. Since, on the other hand, the reduced density $\mathcal{F}_{0}\left(t^{\prime}\right)$ is expressed in terms of the one-fermion densities alone, we see that the resulting equations are now essentially closed equations. Note, however, that the complicated time dependence of the field operators is explicitly probed through the memory effects present in the expression (25) for $\mathcal{F}^{\prime}(t)$. Approximations are, therefore, needed for the actual evaluation of this object. A systematic expansion scheme for the memory effects has been discussed in Refs. $[3,9,10]$. The lowest-order correlation corrections to the pure mean-field approximation, in which $\mathcal{F}^{\prime}$ is simply ignored, correspond to replacing the full Heisenberg time evolution of operators occurring in the collision integrals by a mean-field evolution governed by

$$
H_{0}=\mathcal{P}^{\dagger}(t) H \text {. }
$$

Consistently, with this approximation, $\mathcal{L}$ is replaced in Eqs. (25) and (26) by $\mathcal{L}_{0} \cdot=\left[H_{0}, \cdot\right]$. In this way correlation effects are treated to second order in $H$ in the resulting collision integrals.

An important feature of this scheme (which holds also for higher orders of the expansion [9]) is that the mean energy is conserved, namely,

$$
\frac{\partial}{\partial t}\langle H\rangle=0
$$

where

$$
\langle H\rangle=\operatorname{Tr} H \mathcal{F}_{0}(t)+\operatorname{Tr} H \mathcal{F}^{\prime}(t) .
$$

In the following sections we apply the general expressions obtained above to treat a uniform fermion system described by chiral Gross-Neveu model (CGNM). We will consider only the lowest (mean-field) approximation, corresponding to $\mathcal{F}^{\prime}(t)=0$. Collisional correlations will be treated elsewhere.

\section{THE CHIRAL GROSS-NEVEU MODEL (CGNM)}

The Hamiltonian density for the CGNM is given by

$$
\begin{aligned}
\mathcal{H}_{\mathrm{CGNM}}= & \sum_{i=1}^{N}\left\{\bar{\psi}^{i}\left[-i \gamma_{1} \partial_{1}\right] \psi^{i}\right\} \\
& -\frac{g^{2}}{2}\left\{\left[\sum_{i=1}^{N} \bar{\psi}^{i} \psi^{i}\right]^{2}-\xi\left[\sum_{i=1}^{N} \bar{\psi}^{i} \gamma_{5} \psi^{i}\right]^{2}\right\},
\end{aligned}
$$

where $\xi$ is a constant which indicates whether the model is invariant under the discrete $\gamma_{5}$ transformation $(\xi=0)$ or under the Abelian chiral $\mathrm{U}(1)$ group $(\xi=1)$.
This is a massless fermion theory in $1+1$ dimensions with quartic interaction. The model contains $N$ species of fermions coupled symmetrically, where $\psi^{i}$ is a complex Dirac spinor transforming as the fundamental representation of SU $(N)$ group. It is known that the actual symmetry of the theory is not $\mathrm{SU}(N)$ but rather $\mathrm{O}(2 N)$ [14]. The transformations forming this group mix not only particles but also particles with antiparticles. This model is essentially equivalent to the Nambu-Jona-Lasinio model [15], except for the fact that in $1+1$ dimensions it is renormalizable. Moreover, it is one of the very few known field theories which are asymptotically free. To leading order in a $1 / N$ expansion [11], the CGNM exhibits a number of interesting phenomena, such as spontaneous symmetry breaking [16], dynamical fermion mass generation and dimensional transmutation. The model possesses an infinite number of conservation laws, and as a consequence, the $S$ matrix may be computed exactly [17].

To obtain the time evolution of Bogolyubov parameters we have to obtain the CGNM Hamiltonian [see Eq. (16)] by integrating over one-dimensional space. This involves, in particular, choosing a representation for the $\gamma$ matrices. Here, we have to be careful, since a bad choice of representation can spoil manifest reflection invariance. In Appendix A (see also Ref. [15]) we discuss suitable representations for the $\gamma$ matrices. We choose the Pauli-Dirac representation, namely,

$$
\gamma_{0}=\sigma_{3}, \quad \gamma_{1}=i \sigma_{2},
$$

and

$$
\gamma_{5}=\gamma_{0} \gamma_{1}=\sigma_{1} .
$$

In this representation the spinors $u_{1}(\mathbf{k})$ and $u_{2}(\mathbf{k})$ are given by

$$
\begin{aligned}
& u_{1}(\mathbf{k})=\left[\frac{\left(k_{0}+m\right)}{2 m}\right]^{1 / 2}\left[\begin{array}{c}
1 \\
\frac{\mathbf{k}}{\left(k_{0}+m\right)}
\end{array}\right], \\
& u_{2}(\mathbf{k})=\left[\frac{\left(k_{0}-m\right)}{2 m}\right]^{1 / 2}\left[\begin{array}{c}
1 \\
\frac{\mathbf{k}}{\left(k_{0}-m\right)}
\end{array}\right] .
\end{aligned}
$$

The resulting form for the CGNM Hamiltonian obtained using this representation is given in full in Appendix B.

We next consider the initial-condition problem for this system. The general Bogolyubov transformation defined in Eq. (11) breaks both chiral and charge symmetries, but we restrict the following development to a special Bogolyubov transformation (to be called the Nambu transformation) which breaks the chiral symmetry of our system only. The elements of this Nambu transformation, parametrized consistently with the unitarity conditions (9), are given by

$$
\begin{gathered}
X_{11}=X_{22}=\cos \varphi_{\mathbf{k}} \quad \text { and } \quad X_{12}=X_{21}=0, \\
Y_{12}=-Y_{21}=\sin \varphi_{\mathbf{k}} e^{i \gamma_{\mathbf{k}}} \text { and } \quad Y_{11}=Y_{22}=0 .
\end{gathered}
$$


In the special case of the Nambu transformation, the elements of the matrices $h_{\mathbf{k}}$ and $g_{\mathbf{k}}$ [see Eq. (15)] are given by

$$
\begin{gathered}
h_{11}=h_{22}=\dot{\gamma}_{\mathbf{k}} \sin ^{2} \varphi_{\mathbf{k}}, \\
h_{12}=h_{21}=g_{11}=g_{22}=0, \\
g_{12}=-g_{21}=\left[i \dot{\varphi}_{\mathbf{k}}-\dot{\gamma}_{\mathbf{k}} \sin \varphi_{\mathbf{k}} \cos \varphi_{\mathbf{k}}\right] e^{i \gamma_{\mathbf{k}}} .
\end{gathered}
$$

On the other hand, in the mean-field approximation one finds that $\mathcal{F}_{0}(t)$ commutes with number operators

$$
\operatorname{Tr}\left\{H_{\mathrm{CGNM}}\left[\mathcal{F}_{0}, \beta_{\mathbf{k}, 1}^{\dagger} \beta_{\mathbf{k}, 1}\right]\right\}=\operatorname{Tr}\left\{H_{\mathrm{CGNM}}\left[\mathcal{F}_{0}, \beta_{\mathbf{k}, 2}^{\dagger} \beta_{\mathbf{k}, 2}\right]\right\}=0,
$$

while, due to the charge conservation in the system, we have also

$$
\begin{aligned}
\operatorname{Tr}\left\{\left[\beta_{\mathbf{k}, 1}^{\dagger} \beta_{\mathbf{k}, 2}, H_{\mathrm{CGNM}}\right] \mathcal{F}_{0}\right\} & =\operatorname{Tr}\left\{\left[\beta_{\mathbf{k}, 2}^{\dagger} \beta_{\mathbf{k}, 1}, H_{\mathrm{CGNM}}\right] \mathcal{F}_{0}\right\}=0 \\
\operatorname{Tr}\left\{\left[\beta_{-\mathbf{k}, 1} \beta_{\mathbf{k}, 1}, H_{\mathrm{CGNM}}\right] \mathcal{F}_{0}\right\} & =\operatorname{Tr}\left\{\left[\beta_{-\mathbf{k}, 2} \beta_{\mathbf{k}, 2}, H_{\mathrm{CGNM}}\right] \mathcal{F}_{0}\right\} \\
& =0 .
\end{aligned}
$$

Substituting Eqs. (31)-(33) in Eq. (16), we obtain the equations which describe the time evolution of our system as

$$
\begin{gathered}
\dot{\nu}_{\mathbf{k}, 1}=0 \quad \text { and } \quad \dot{\nu}_{\mathbf{k}, 2}=0, \\
{\left[i \dot{\varphi}_{\mathbf{k}}+\dot{\gamma}_{\mathbf{k}} \sin \varphi_{\mathbf{k}} \cos \varphi_{\mathbf{k}}\right] e^{-i \gamma_{\mathbf{k}}}} \\
=\frac{\operatorname{Tr}\left(\left[\beta_{-\mathbf{k}, 1} \beta_{\mathbf{k}, 2}, H_{\mathrm{CGNM}}\right] \mathcal{F}_{0}\right)}{\left(1-\nu_{\mathbf{k}, 1}-\nu_{\mathbf{k}, 2}\right)} .
\end{gathered}
$$

Equation (34) shows that the occupation numbers of the paired natural orbitals are constant, i.e., we recover the general isoentropic character of the mean-field approximation. The complex equation of motion (35) describes the time evolution of the Nambu parameters. Writing the CGNM Hamiltonian, given in Appendix B, in the Nambu basis using Eqs. (11) and (30), and substituting this Hamiltonian in Eq. (35), we obtain the explicit form of this equation. The calculation of traces is lengthy but straightforward. As a result, one obtains

$$
\begin{aligned}
& i \dot{\varphi}_{\mathbf{k}}+\dot{\gamma}_{\mathbf{k}} \frac{\sin 2 \varphi_{\mathbf{k}}}{2} \\
& =\frac{(\mathbf{k})^{2}}{k_{0}} \sin 2 \varphi_{\mathbf{k}}-m \frac{|\mathbf{k}|}{k_{0}}\left[\sin ^{2} \varphi_{\mathbf{k}} e^{\left.-i \gamma_{\mathbf{k}}-\cos ^{2} \varphi_{\mathbf{k}} e^{i \gamma_{\mathbf{k}}}\right]}\right. \\
& +\left(\frac{g^{2} m^{2}}{4 \pi}\right) \frac{(\xi+1)}{k_{0}} \\
& \times\left[\sin 2 \varphi_{\mathbf{k}}+\frac{|\mathbf{k}|}{m}\left(\sin ^{2} \varphi_{\mathbf{k}} e^{-i \gamma_{\mathbf{k}}}-\cos ^{2} \varphi_{\mathbf{k}} e^{i \gamma_{\mathbf{k}}}\right)\right]\left(I_{1}+I_{2}\right),
\end{aligned}
$$

where $I_{1}$ and $I_{2}$ are the divergent integrals

$$
\begin{gathered}
I_{1}=\int \frac{d \mathbf{k}^{\prime}}{k_{0}^{\prime}} \cos 2 \varphi_{\mathbf{k}^{\prime}}\left(1-\nu_{\mathbf{k}^{\prime}, 1}-\nu_{\mathbf{k}^{\prime}, 2}\right), \\
I_{2}=\int \frac{d \mathbf{k}^{\prime}}{k_{0}^{\prime}} \frac{\left|\mathbf{k}^{\prime}\right|}{m} \sin 2 \varphi_{\mathbf{k}^{\prime}} \cos \gamma_{\mathbf{k}^{\prime}}\left(1-\nu_{\mathbf{k}^{\prime}, 1}-\nu_{\mathbf{k}^{\prime}, 2}\right) .
\end{gathered}
$$

We take $N=1$ for simplicity. Splitting the complex equation (36) into real and imaginary parts, we have

$$
\begin{aligned}
&\left.\dot{\varphi}_{\mathbf{k}}=\sin \gamma_{\mathbf{k}} \frac{|\mathbf{k}|}{k_{0}} \mid m-\left(\frac{g^{2} m}{4 \pi}\right)(\xi+1)\left(I_{1}+I_{2}\right)\right], \\
& \dot{\gamma}_{\mathbf{k}} \sin 2 \varphi_{\mathbf{k}}= \frac{2 \sin 2 \varphi_{\mathbf{k}}}{k_{0}}\left[\mathbf{k}^{2}+\left(\frac{g^{2} m^{2}}{4 \pi}\right)(\xi+1)\left(I_{1}+I_{2}\right)\right] \\
&+2 \cos 2 \varphi_{\mathbf{k}} \cos \gamma_{\mathbf{k}} \frac{|\mathbf{k}|}{k_{0}} \\
& \times {\left[m-\left(\frac{g^{2} m}{4 \pi}\right)(\xi+1)\left(I_{1}+I_{2}\right)\right] . }
\end{aligned}
$$

Finally, the mean-field energy is evaluated as

$$
\begin{aligned}
\left\langle H_{\mathrm{CGNM}}^{\mathrm{MF}}\right\rangle= & \operatorname{Tr}\left[H_{\mathrm{CGNM}} \mathcal{F}_{0}(t)\right] \\
= & \left(\frac{m^{2}}{2 \pi}\right)\left(I_{2}-I_{3}\right)-\left(\frac{g^{2} m^{2}}{8 \pi^{2}}\right) \frac{(\xi+1)}{2}\left(I_{1}+I_{2}\right)^{2} \\
& -\left(\frac{g^{2}}{8 \pi^{2}}\right) \frac{(\xi+1)}{2} \int d \mathbf{k}^{\prime} \\
& \times\left(1+\nu_{\mathbf{k}^{\prime}, 1}-\nu_{\mathbf{k}^{\prime}, 2}\right) \int d \mathbf{k}^{\prime \prime}\left(1-\nu_{\mathbf{k}^{\prime \prime}, 1}+\nu_{\mathbf{k}^{\prime \prime}, 2}\right),
\end{aligned}
$$

where $I_{1}$ and $I_{2}$ are given in Eq. (37) and $I_{3}$ is given by

$$
I_{3}=\int \frac{d \mathbf{k}^{\prime}}{k_{0}^{\prime}}\left(\frac{\mathbf{k}^{\prime}}{m}\right)^{2} \cos 2 \varphi_{\mathbf{k}}\left(1-\nu_{\mathbf{k}^{\prime}, 1}-\nu_{\mathbf{k}^{\prime}, 2}\right) .
$$

Since all the results above contain divergent integrals, a renormalization procedure is required. This is discussed in the next section.

\section{RENORMALIZATION}

In order to handle the infinities which occur in the preceding equations it is necessary to introduce a renormalization prescription that will render physical quantities finite. In general, renormalization procedures consist in combining divergent terms with the bare mass and coupling constants of the theory to define new, finite (or renormalized) values of these quantities. Thus, when one adopts a momentum cutoff to regularize divergent integrals, the bare mass and coupling constants are chosen to be cutoff dependent in a way that will cancel the divergences. In the present case, however, the divergent integrals (37) and (40) involve the dynamical variables themselves in the integrand, so that even their degree of divergence is not directly computable. In order to handle this situation we will use a self-consistent renormalization procedure inspired by Ref. [18]. 
The renormalization prescription we use can be based on the consideration of the static solutions of the dynamical equations (38). They are determined by the equations

$$
\begin{gathered}
\left.\sin \gamma_{\mathbf{k}}\right|_{\mathrm{eq}}\left[1-\left(\frac{g^{2}}{4 \pi}\right)(\xi+1)\left(I_{1}+I_{2}\right)\right]=0 \\
\left.\tan 2 \varphi_{\mathbf{k}}\right|_{\mathrm{eq}}=\left.\frac{-|\mathbf{k}| m\left[1-\left(g^{2} / 4 \pi\right)(\xi+1)\left(I_{1}+I_{2}\right)\right]}{\left[(\mathbf{k})^{2}+\left(g^{2} m^{2} / 4 \pi\right)(\xi+1)\left(I_{1}+I_{2}\right)\right]} \cos \gamma_{\mathbf{k}}\right|_{\mathrm{eq}} .
\end{gathered}
$$

We will then show explicitly that it also controls the divergences which appear in the kinetic regime of the mean-field approximation.

In order to obtain the renormalization prescription we introduce a momentum cutoff $\Lambda$ and begin by assuming that, in order to render the theory finite, the bare coupling constant $g^{2}$ must approach zero for large values of $\Lambda$ as (see, e.g., Refs. $[11,19])$

$$
g^{2}=\frac{4 \pi}{(\xi+1)}\left[\ln \left(\frac{\Lambda^{2}}{m^{2}}\right)\right]^{-1},
$$

where the form of the first factor is dictated by later convenience. We next assume that the integrals $I_{1}$ and $I_{2}$ have logarithmic divergences

$$
\begin{aligned}
& I_{1}=a+b \ln \left(\frac{\Lambda^{2}}{m^{2}}\right), \\
& I_{2}=c+d \ln \left(\frac{\Lambda^{2}}{m^{2}}\right),
\end{aligned}
$$

where $a, b, c$, and $d$ are finite constants. Substituting Eq. (43) and the Ansätze (44) and (45) in the static equation (42), we obtain

$$
\left.\tan 2 \varphi_{\mathbf{k}}\right|_{\mathrm{eq}}=\frac{-(-1)^{n} m|\mathbf{k}|[1-(b+d)]}{\left[(\mathbf{k})^{2}+m^{2}(b+d)\right]} .
$$

Finally, evaluating the integrals $I_{1}$ and $I_{2}$, Eqs. (37), using Eq. (46), we are able to determine the constants $a, b, c$, and $d$ self-consistently. This calculation is given in Appendix $\mathrm{C}$. We find there $b=1$ while $d$ remains arbitrary. The renormalized static equations are then obtained by simply substituting these values in Eq. (46). We obtain

$$
\left.\tan 2 \varphi_{\mathbf{k}}\right|_{\mathrm{eq}}=\frac{(-1)^{n} m|\mathbf{k}| d}{\left[\mathbf{k}^{2}+(1+d) m^{2}\right]} .
$$

We observe that the theory involves just one free parameter $d$. This is altogether reasonable since our starting point was a massless fermion theory which was determined by one dimensionless coupling constant $g^{2}$, and we end up with a theory determined by one free parameter $d$ after the selfconsistent renormalization procedure. What is needed is an interpretation of the parameter $d$.

To obtain this, we begin by writing the fields $\psi(\mathbf{x})$ and $\bar{\psi}(\mathbf{x})$ given in Eq. (1) in the Nambu quasiparticle basis using Eqs. (11) and (30). We find that the new Dirac spinors in this basis are given by

$$
\begin{gathered}
u_{1}^{\prime}(\mathbf{k})=\cos \varphi_{\mathbf{k}} u_{1}(\mathbf{k})+\sin \varphi_{\mathbf{k}} e^{i \gamma_{\mathbf{k}}} u_{2}(-\mathbf{k}), \\
u_{2}^{\prime}(\mathbf{k})=\cos \varphi_{\mathbf{k}} u_{2}(\mathbf{k})-\sin \varphi_{\mathbf{k}} e^{-i \gamma_{\mathbf{k}}} u_{1}(-\mathbf{k})
\end{gathered}
$$

From the renormalized static solution (47) we have that

$$
\begin{aligned}
\left.\cos \varphi_{\mathbf{k}}\right|_{\text {eq }}=\frac{1}{2 \sqrt{k_{0} x}} & {\left[\left(k_{0} x+m|\mathbf{k}| d\right)^{1 / 2}+\left(k_{0} x-m|\mathbf{k}| d\right)^{1 / 2}\right], } \\
\left.\sin \varphi_{\mathbf{k}}\right|_{\mathrm{eq}}= & (-1)^{n} \frac{1}{2 \sqrt{k_{0} x}}\left[\left(k_{0} x+m|\mathbf{k}| d\right)^{1 / 2}\right. \\
& \left.-\left(k_{0} x-m|\mathbf{k}| d\right)^{1 / 2}\right]
\end{aligned}
$$

where $x=\left[k^{2}+(1+d)^{2} m^{2}\right]^{1 / 2}$. Substituting the solutions (49) and the particle spinors (29) in Eq. (48), we obtain the new spinors in quasiparticle basis. They are given as

$$
u_{1}^{\prime}(\mathbf{k})=\left[\frac{\left(k_{0}^{\mathrm{eff}}+m_{\mathrm{eff}}\right)}{2 m_{\mathrm{eff}}}\right]^{1 / 2}\left[\begin{array}{c}
1 \\
\frac{\mathbf{k}}{\left(k_{0}^{\mathrm{eff}}+m_{\mathrm{eff}}\right)}
\end{array}\right],
$$

$$
u_{2}^{\prime}(\mathbf{k})=\left[\frac{\left(k_{0}^{\mathrm{eff}}-m_{\mathrm{eff}}\right)}{2 m_{\mathrm{eff}}}\right]^{1 / 2}\left[\begin{array}{c}
1 \\
\frac{\mathbf{k}}{\left(k_{0}^{\mathrm{eff}}-m_{\mathrm{eff}}\right)}
\end{array}\right],
$$

where

$$
\left(k_{0}^{\mathrm{eff}}\right)^{2}=(\mathbf{k})^{2}+\left(m_{\mathrm{eff}}\right)^{2} \quad \text { and } \quad m_{\mathrm{eff}}=(1+d) m .
$$

Comparing the spinors (50) in quasiparticle basis with the spinors (29) in particle basis, we see in Eq. (51) what amounts to a redefinition of the mass scale. Therefore, we note that, unlike the situation found in connection with the $1 / N$ expansion, the use of the Gaussian Ansatz, Eq. (19), parametrized by the canonical transformation leading to the quasifermion basis, allows for the direct dynamical determination of the stable equilibrium situation of the system [see Eqs. (41) and (42)], including symmetry breaking and mass generation. Moreover, the renormalization procedure effectively replaces the dimensionless coupling constant $g^{2}$ by the free parameter $d$ associated to the mass scale [see Eq. (51)]. This is analogous to the phenomenon of dimensional transmutation found by Gross and Neveu [11] in the $1 / N$ expansion. Finally, aside from the overall mass scale (characterized by $d$ ) there are no free adjustable parameters.

Using Eqs. (38), (43)-(45), we finally write the renormalized form of the dynamical equations that describe the meanfield time evolution of this system. As mentioned before, they are now also finite and read 


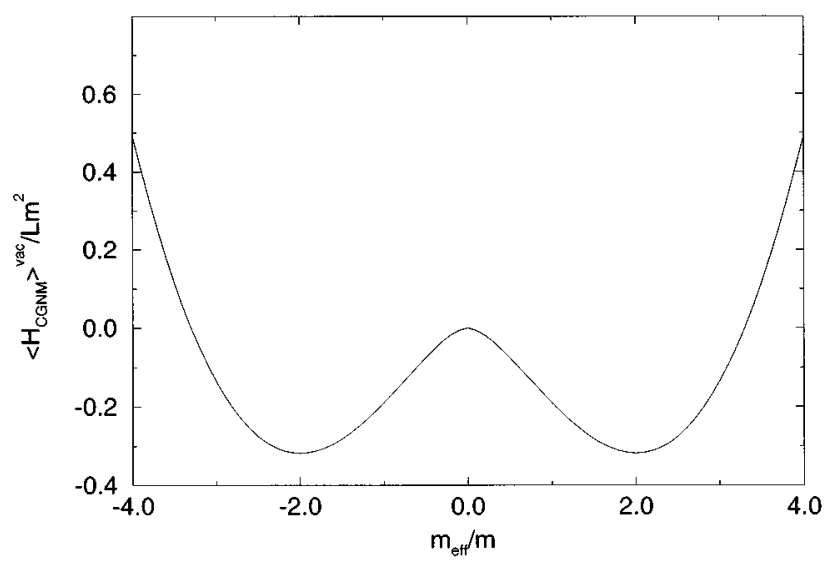

FIG. 1. Mean-field effective potential for the ground state of a uniform fermion system described by the CGNM as function of effective mass $m_{\text {eff }}$.

$$
\begin{gathered}
\dot{\nu}_{\mathbf{k}, 1}=\dot{\nu}_{\mathbf{k}, 2}=0, \\
\dot{\varphi}_{\mathbf{k}}=-m d \frac{|\mathbf{k}|}{k_{0}} \sin \gamma_{\mathbf{k}}, \\
\dot{\gamma}_{\mathbf{k}} \sin 2 \varphi_{\mathbf{k}}=\frac{2 \sin 2 \varphi_{\mathbf{k}}}{k_{0}}\left[(\mathbf{k})^{2}+m^{2}(1+d)\right] \\
-2 m d \frac{|\mathbf{k}|}{k_{0}} \cos 2 \varphi_{\mathbf{k}} \cos \gamma_{\mathbf{k}} .
\end{gathered}
$$

The first two equations express just the isoentropic character of the mean-field kinetics. The remaining coupled equations describe the kinetic behavior of the Nambu parameters and are discussed in the following section.

We conclude this section calculating the ground-state (vacuum) energy of our system in the mean-field approximation. This approximation to the ground state is obtained as a static solution of Eqs. (52). The associated mean energy can be obtained by taking $\nu_{\mathbf{k}, 1}=\nu_{\mathbf{k}, 2}=0$ in Eq. (39) and evaluating the divergent integrals (37) and (40) using the renormalization Ansätze (43), (44), and (45). We obtain

$$
\begin{aligned}
\frac{\left\langle H_{\mathrm{CGNM}}^{\mathrm{MF}}\right\rangle^{\text {vacuum }}}{L}= & -\left(\frac{1}{2 \pi}\right)\left[1+\left(\frac{g^{2}}{2 \pi}\right)(\xi+1)\right] \Lambda^{2} \\
& -\left(\frac{1}{2 \pi}\right) \frac{(1+d)^{2} m^{2}}{2}\left\{1-\ln \left[\frac{(1+d)^{2}}{4}\right]\right\},
\end{aligned}
$$

where we used for the finite constants $a, b$, and $c$ the expresions given in Appendix $\mathrm{C}$. The first term, divergent as $\Lambda \rightarrow \infty$, represents a vacuum background energy. The presence of this term has no physical consequences, and we follow the usual practice of redefining the zero of the energy scale by simply subtracting it out. Therefore, in terms of the effective mass given by Eq. (51), the renormalized energy density of the ground state of our system is given by

$$
\frac{\left\langle H_{\mathrm{CGNM}}^{\mathrm{MF}}\right\rangle^{\text {vacuum }}}{L}=-\left(\frac{m^{2}}{4 \pi}\right)\left(\frac{m_{\mathrm{eff}}}{m}\right)^{2}\left[1+2 \ln 2-\ln \left(\frac{m_{\mathrm{eff}}}{m}\right)^{2}\right] .
$$

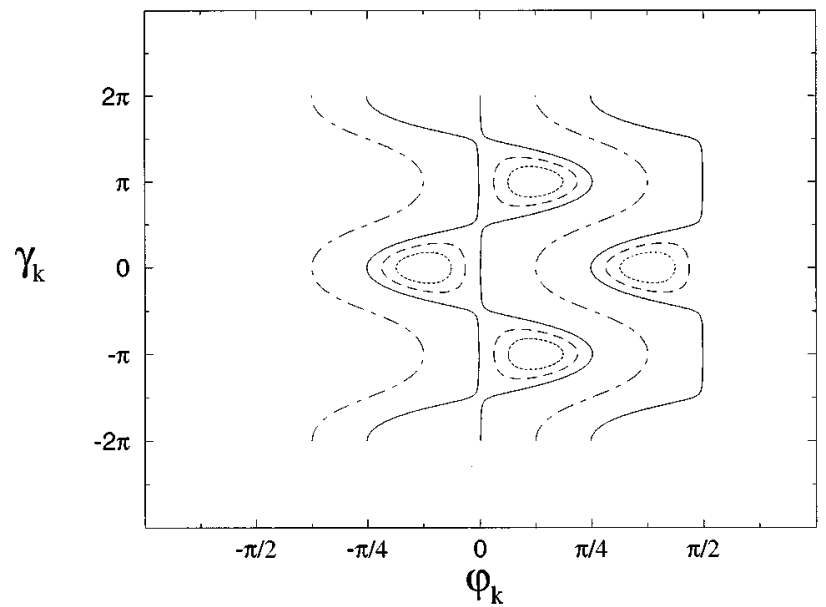

FIG. 2. Phase-space of Nambu parameters for $|\mathbf{k}|=m=\sqrt{2}$ in the case of free fermion system $\left[(d+1=0)\right.$ or $\left.m_{\mathrm{eff}}=0\right]$. Initial values: Dotted line: $\varphi_{\mathbf{k}}^{\text {in }}=(-0.2+n \pi / 2), \quad \gamma_{\mathbf{k}}^{\text {in }}=2 n \pi$ and $\varphi_{\mathbf{k}}^{\text {in }}=(+0.2+n \pi / 2), \quad \gamma_{\mathbf{k}}^{\text {in }}=(2 n+1) \pi ;$ Dashed line: $\varphi_{\mathbf{k}}^{\text {in }}=(-0.1$ $+n \pi / 2), \quad \gamma_{\mathbf{k}}^{\text {in }}=2 n \pi \quad$ and $\quad \varphi_{\mathbf{k}}^{\text {in }}=(+0.1+n \pi / 2), \quad \gamma_{\mathbf{k}}^{\text {in }}=(2 n+1) \pi ;$ Solid line: $\varphi_{\mathbf{k}}^{\text {in }}=(-\pi / 4+n \pi / 2), \quad \gamma_{\mathbf{k}}^{\text {in }}=0$ and $\varphi_{\mathbf{k}}^{\text {in }}=(0+n \pi / 2)$, $\gamma_{\mathbf{k}}^{\text {in }}=0$; Dot-dashed line: $\varphi_{\mathbf{k}}^{\text {in }}=(\pi / 8+n \pi / 2), \quad \gamma_{\mathbf{k}}^{\text {in }}=0$.

Figure 1 shows the renormalized ground-state energy density as a function of effective mass $m_{\text {eff }}$. This figure reproduces the well-known effective potential obtained in the case of $1 / N$ expansion [11].

\section{KINETICS OF ONE-FERMION DENSITIES}

In this section, we discuss the solutions of the nonlinear equations of motion of the Nambu parameters $\varphi_{\mathbf{k}}$ and $\gamma_{\mathbf{k}}$ in terms of the values taken by the parameter $d$. In order to do this, it is useful to note that these equations can be obtained as the canonical equations of the effective $c$-number Hamiltonian

$$
h_{\mathrm{eff}}=\frac{2}{k_{0}}\left[\mathbf{k}^{2}+m^{2}(1+d)\right] \sigma_{\mathbf{k}}-\frac{2 m|\mathbf{k}|}{k_{0}} d \cos \gamma_{\mathbf{k}}\left(1-\sigma_{\mathbf{k}}^{2}\right)^{1 / 2} \text {, }
$$

where we defined $\sigma_{\mathbf{k}}=-\cos 2 \varphi_{\mathbf{k}}$ as a new momentumlike variable canonically conjugate to $\gamma_{\mathbf{k}}$. For a general value of $d$ this dynamical system has equilibrium solutions given by $\left.\gamma_{\mathbf{k}}\right|_{\text {eq }}=n \pi$ with $\left.\varphi_{\mathbf{k}}\right|_{\text {eq }}$ given by Eq. (47). A special situation occurs, however, when $d=0$. In this case, in fact, $\varphi_{\mathbf{k}}$ becomes a constant of motion ( $\gamma_{\mathbf{k}}$ becomes cyclical in $\left.h_{\text {eff }}\right)$ and $\dot{\gamma}_{\mathbf{k}}$ becomes independent of $\varphi_{\mathbf{k}}$.

Considering next the time-dependent solutions in the general case $(d \neq 0)$, we see that the equilibrium solutions are stable in the sense that small initial displacements from the equilibrium states lead to librational behavior both in $\varphi_{\mathbf{k}}$ and in $\gamma_{\mathbf{k}}$. For sufficiently large displacements, however, the latter variable acquires a rotational (though nonuniform) behavior while $\varphi_{\mathbf{k}}\left(\right.$ or $\sigma_{\mathbf{k}}$ ) still oscillates about an equilibrium value. A set of phase-space trajectories illustrating this behavior is shown in Figs. 2 and 3. As one sees in these figures, when $d$ is closer to the special value zero, the stability domains shrink in the variable $\varphi_{\mathbf{k}}$ as the equilibrium points approach the lines $\varphi_{\mathbf{k}}=m \pi / 2$. If, on the other hand, one 


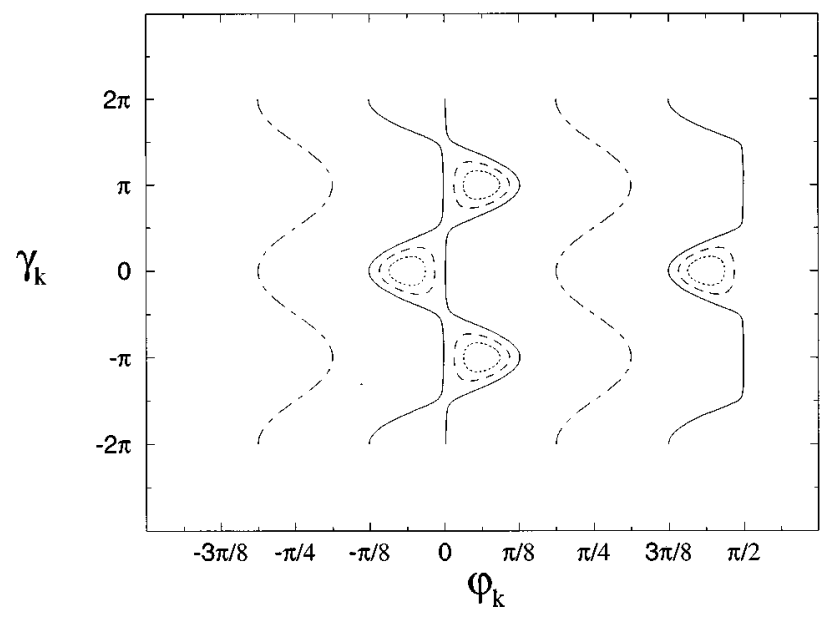

FIG. 3. Same as Fig. 2 with $(d+1)=0.5858$. Initial values: Dotted line: $\varphi_{\mathbf{k}}^{\text {in }}=(-0.1+n \pi / 2), \quad \gamma_{\mathbf{k}}^{\text {in }}=2 n \pi \quad$ and $\quad \varphi_{\mathbf{k}}^{\text {in }}=(+0.1$ $+n \pi / 2), \quad \gamma_{\mathbf{k}}^{\text {in }}=(2 n+1) \pi ; \quad$ Dashed line: $\varphi_{\mathbf{k}}^{\text {in }}=(-0.05+n \pi / 2)$, $\gamma_{\mathbf{k}}^{\text {in }}=2 n \pi$ and $\varphi_{\mathbf{k}}^{\text {in }}=(+0.05+n \pi / 2), \quad \gamma_{\mathbf{k}}^{\text {in }}=(2 n+1) \pi$; Solid line: $\varphi_{\mathbf{k}}^{\text {in }}=(-\pi / 8+n \pi / 2), \quad \gamma_{\mathbf{k}}^{\text {in }}=0$ and $\varphi_{\mathbf{k}}^{\text {in }}=(0+n \pi / 2), \quad \gamma_{\mathbf{k}}^{\text {in }}=0$; Dotdashed line: $\varphi_{\mathbf{k}}^{\text {in }}=(3 \pi / 16+n \pi / 2), \quad \gamma_{\mathbf{k}}^{\text {in }}=0$.

considers the kinetic equations for $d=0$, one sees that the solutions correspond to uniform $\gamma_{\mathbf{k}}$ rotations with fixed $\varphi_{\mathbf{k}}$.

In order to interpret this behavior we recall that $d$, together with the expansion mass $m$, and the equilibrium values of $\varphi_{\mathbf{k}}$ and $\gamma_{\mathbf{k}}$, define the equilibrium effective mass $m_{\text {eff }}$ [see Eq. (51)]. In particular, when $d=-1$, one has $m_{\mathrm{eff}}=0$ and the equations of motion reduce to

$$
\begin{gathered}
\dot{\varphi}_{\mathbf{k}}=m \frac{|\mathbf{k}|}{k_{0}} \sin \gamma_{\mathbf{k}}, \\
\dot{\gamma}_{\mathbf{k}} \sin 2 \varphi_{\mathbf{k}}=2 \frac{(\mathbf{k})^{2}}{k_{0}} \sin 2 \varphi_{\mathbf{k}}+2 m \frac{|\mathbf{k}|}{k_{0}} \cos 2 \varphi_{\mathbf{k}} \cos \gamma_{\mathbf{k}},
\end{gathered}
$$

which reproduce the equations one obtains for a free (massless) field, e.g., by making $g=0$ in the Hamiltonian density (27). Other values of $d$ will, on the other hand, correspond to nonvanishing equilibrium values of the effective mass. Displacements of $\varphi_{\mathbf{k}}$ and $\gamma_{\mathbf{k}}$ from their respective equilibrium values will then correspond to preparing the system in a state having a "wrong" (k-dependent) effective mass (in particular, nonvanishing in the "symmetric" phase, $d=-1$ ). The ensuing motion represents, therefore, the dynamical reaction of the system to this situation. Several phase-space trajectories for off-equilibrium solutions are shown in Figs. 2 and 3.

The special case $d=0$ corresponds to choosing the equilibrium effective mass $m_{\text {eff }}$ itself as the expansion mass $m$. In this case the stability islands disappear, as the phase-space trajectories degenerate to straight lines. The off-equilibrium dynamics can then be represented by the time dependence of the single parameter $\gamma_{\mathbf{k}}$.

\section{DISCUSSION AND CONCLUSIONS}

We have described a treatment of the initial-value problem in a quantum-field theory of self-interacting fermions in the Gaussian approximation. Although the procedure is quite general, we have implemented it for the vacuum of a relativ- istic many-fermion system described by the chiral GrossNeveu model (CGNM).

We have obtained the renormalized kinetic equations which describe the effective dynamics of Gaussian observables in the mean-field approximation for a uniform $(1+1)$-dimensional system. We used the static solution of these equations in order to renormalize the theory, leading to an effective potential similar to that obtained by Gross and Neveu using the $1 / N$ expansion. We show also that other static results discussed in the literature, such as dynamical mass generation due to chiral symmetry breaking and a phenomenon analogous to dimensional transmutation, can be retrieved from this formulation in the mean-field approximation. Finally, we obtained and discussed numerical solutions for the time evolution of the Nambu parameters (Gaussian variables) for nonequilibrium initial conditions.

As a final comment we note that, unlike the situation found in connection with the $1 / N$ expansion, the use of the Gaussian Ansatz, Eq. (19), parametrized by the canonical transformation leading to the quasifermion basis, allows for the direct dynamical determination of the stable equilibrium situations of the system [see Eqs. (41) and (42)], with or without symmetry breaking and mass generation. In the latter case we obtain just a free, massless theory.

The present projection technique [8] can be extended to include and to evaluate higher dynamical correlation corrections to the mean-field approximation. In this case the occupation numbers are no longer constant, $\dot{v}_{\mathbf{k}, \lambda} \neq 0$, and their time dependence affects the effective dynamics of the Gaussian variables (see Ref. [10]). A finite-matter density calculation beyond the mean-field approximation allows one to study collisional observables such as transport coefficients [20]. Finally, we comment on the extension to nonuniform systems. In this case the spatial dependence of the fields $\bar{\psi}(x)$ and $\psi(x)$ are expanded in the natural orbitals through the use of a nonhomogeneous Bogolyubov transformation (see Ref. [9]).

\section{ACKNOWLEDGMENTS}

One of the authors (P.L.N.) was supported by Conselho Nacional de Desenvolvimento Científico e Tecnológico (CNPq), Brazil; and by Fundação de Amparo à Pesquisa do Estado de São Paulo (FAPESP), Brazil.

\section{APPENDIX A: UNIFORM SYSTEM AND THE REPRESENTATIONS FOR THE $\gamma^{\mu}$ MATRICES}

A (1+1)-dimensional uniform system has to be invariant under translations and under reflection. Reflection invariance implies that the equations of motion are direction independent, i.e., dynamical quantities should involve $|\mathbf{k}|$ only.

Let us consider the solutions of the Dirac equations

$$
\left(i \partial l-m_{j}\right) \psi^{\left(m_{j}\right)}(x)=0, \quad j=1,2,
$$

for two different values $m_{1}$ and $m_{2}$ of the mass:

$$
\begin{aligned}
\psi^{\left(m_{1}\right)}(x)= & \sum_{\mathbf{k}}\left(\frac{m_{1}}{L k_{0}^{\left(m_{1}\right)}}\right)^{1 / 2}\left[b_{\mathbf{k}, 1}^{\left(m_{1}\right)} u_{1}^{\left(m_{1}\right)}(\mathbf{k}) e^{i \mathbf{k} \cdot \mathbf{x}}\right. \\
& \left.+b_{\mathbf{k}, 2}^{\left(m_{1}\right) \dagger} u_{2}^{\left(m_{1}\right)}(\mathbf{k}) e^{-i \mathbf{k} \cdot \mathbf{x}}\right],
\end{aligned}
$$




$$
\begin{aligned}
\bar{\psi}^{\left(m_{1}\right)}(x)= & \sum_{\mathbf{k}}\left(\frac{m_{1}}{L k_{0}^{\left(m_{1}\right)}}\right)^{1 / 2}\left[b_{\mathbf{k}, 1}^{\left(m_{1}\right) \dagger} \bar{u}_{1}^{\left(m_{1}\right)}(\mathbf{k}) e^{-i \mathbf{k} \cdot \mathbf{x}}\right. \\
& +b_{\mathbf{k}, 2}^{\left(m_{1}\right)} \bar{u}_{2}^{\left(m_{1}\right)}(\mathbf{k}) e^{i \mathbf{k} \cdot \mathbf{x}}
\end{aligned}
$$

and

$$
\begin{aligned}
\psi^{\left(m_{2}\right)}(x)= & \sum_{\mathbf{k}}\left(\frac{m_{2}}{L k_{0}^{\left(m_{2}\right)}}\right)^{1 / 2}\left[b_{\mathbf{k}, 1}^{\left(m_{2}\right)} u_{1}^{\left(m_{2}\right)}(\mathbf{k}) e^{i \mathbf{k} \cdot \mathbf{x}}\right. \\
& \left.+b_{\mathbf{k}, 2}^{\left(m_{2}\right) \dagger} u_{2}^{\left(m_{2}\right)}(\mathbf{k}) e^{-i \mathbf{k} \cdot \mathbf{x}}\right], \\
\bar{\psi}^{\left(m_{2}\right)}(x)= & \sum_{\mathbf{k}}\left(\frac{m_{2}}{L k_{0}^{\left(m_{2}\right)}}\right)^{1 / 2}\left[b_{\mathbf{k}, 1}^{\left(m_{2}\right) \dagger} \bar{u}_{1}^{\left(m_{2}\right)}(\mathbf{k}) e^{-i \mathbf{k} \cdot \mathbf{x}}\right. \\
& \left.+b_{\mathbf{k}, 2}^{\left(m_{2}\right)} \bar{u}_{2}^{\left(m_{2}\right)}(\mathbf{k}) e^{i \mathbf{k} \cdot \mathbf{x}}\right] .
\end{aligned}
$$

By imposing (say at $t=0$ ) the condition

$$
\psi^{\left(m_{1}\right)}(x)=\psi^{\left(m_{2}\right)}(x),
$$

one can show that the operator sets $\left(b_{\mathbf{k}, 1}^{\left(m_{1}\right)}, b_{\mathbf{k}, 2}^{\left(m_{1}\right)}\right)$ and $\left(b_{\mathbf{k}, 1}^{\left(m_{2}\right)}, b_{\mathbf{k}, 2}^{\left(m_{2}\right)}\right)$ are related by the canonical transformation

$$
\begin{aligned}
b_{\mathbf{k}, 1}^{\left(m_{2}\right)}= & \left\{\left(\frac{m_{1}}{m_{2}}\right)^{1 / 2}\left(\frac{k_{0}^{\left(m_{2}\right)}}{k_{0}^{\left(m_{1}\right)}}\right)^{1 / 2} \vec{u}_{1}^{\left(m_{2}\right)}(\mathbf{k}) u_{1}^{\left(m_{1}\right)}(\mathbf{k})\right\} b_{\mathbf{k}, 1}^{\left(m_{1}\right)} \\
& +\left\{\left(\frac{m_{1}}{m_{2}}\right)^{1 / 2}\left(\frac{k_{0}^{\left(m_{2}\right)}}{k_{0}^{\left(m_{1}\right)}}\right)^{1 / 2} \bar{u}_{1}^{\left(m_{2}\right)}(\mathbf{k}) u_{2}^{\left(m_{1}\right)}(-\mathbf{k})\right\} b_{-\mathbf{k}, 2}^{\left(m_{1}\right)}, \\
b_{\mathbf{k}, 2}^{\left(m_{2}\right)}= & -\left\{\left(\frac{m_{1}}{m_{2}}\right)^{1 / 2}\left(\frac{k_{0}^{\left(m_{2}\right)}}{k_{0}^{\left(m_{1}\right)}}\right)^{1 / 2} \vec{u}_{2}^{\left(m_{1}\right)}(\mathbf{k}) u_{2}^{\left(m_{2}\right)}(\mathbf{k})\right\} b_{\mathbf{k}, 2}^{\left(m_{1}\right)} \\
& -\left\{\left(\frac{m_{1}}{m_{2}}\right)^{1 / 2}\left(\frac{k_{0}^{\left(m_{2}\right)}}{k_{0}^{\left(m_{1}\right)}}\right)^{1 / 2} \vec{u}_{1}^{\left(m_{1}\right)}(-\mathbf{k}) u_{2}^{\left(m_{2}\right)}(\mathbf{k})\right\} b_{-\mathbf{k}, 1}^{\left(m_{1}\right)} .
\end{aligned}
$$

Reflection invariance requires that the coefficients of this canonical transformation be invariant under $\mathbf{k} \rightarrow-\mathbf{k}$ : i.e.,

$$
\begin{aligned}
& \vec{u}_{1}^{\left(m_{2}\right)}(\mathbf{k}) u_{1}^{\left(m_{1}\right)}(\mathbf{k})=\vec{u}_{1}^{\left(m_{2}\right)}(-\mathbf{k}) u_{1}^{\left(m_{1}\right)}(-\mathbf{k}), \\
& \vec{u}_{1}^{\left(m_{2}\right)}(\mathbf{k}) u_{2}^{\left(m_{1}\right)}(-\mathbf{k})=\vec{u}_{1}^{\left(m_{2}\right)}(-\mathbf{k}) u_{2}^{\left(m_{1}\right)}(\mathbf{k}), \\
& \vec{u}_{2}^{\left(m_{1}\right)}(\mathbf{k}) u_{2}^{\left(m_{2}\right)}(\mathbf{k})=\vec{u}_{2}^{\left(m_{1}\right)}(-\mathbf{k}) u_{2}^{\left(m_{2}\right)}(-\mathbf{k}), \\
& \vec{u}_{1}^{\left(m_{1}\right)}(-\mathbf{k}) u_{2}^{\left(m_{2}\right)}(\mathbf{k})=\vec{u}_{1}^{\left(m_{1}\right)}(\mathbf{k}) u_{2}^{\left(m_{2}\right)}(-\mathbf{k}) .
\end{aligned}
$$

There are just two representations of the $\gamma^{\mu}$ matrices satisfying these conditions. These are the Pauli-Dirac representation

$$
\gamma_{0}=\sigma_{3}, \quad \gamma_{1}=i \sigma_{2}, \quad \text { and } \quad \gamma_{5}=\gamma_{0} \gamma_{1}=\sigma_{1}
$$

and

$$
\gamma_{0}=\sigma_{3}, \quad \gamma_{1}=i \sigma_{1}, \quad \text { and } \quad \gamma_{5}=\gamma_{0} \gamma_{1}=-\sigma_{2}
$$

We choose the Pauli-Dirac representation (A10) which admit as solutions the spinors given in Eq. (29). It should be noted, however, that the spinors

$$
\begin{aligned}
& u_{1}(\mathbf{k})=\left(\frac{k_{0}+m}{2 m}\right)^{1 / 2}\left[\begin{array}{c}
1 \\
\frac{\mathbf{k}}{\left(k_{0}+m\right)}
\end{array}\right], \\
& u_{2}(\mathbf{k})=\left(\frac{k_{0}+m}{2 m}\right)^{1 / 2}\left[\begin{array}{c}
\frac{\mathbf{k}}{\left(k_{0}+m\right)} \\
1
\end{array}\right],
\end{aligned}
$$

which are also solutions of Dirac's equation in the PauliDirac representation, do not satisfy the uniformity conditions (A9).

\section{APPENDIX B: THE UNIFORM CGNM HAMILTONIAN}

In this appendix we give explicitly the Hamiltonian of the chiral Gross-Neveu model in a form which preserves the reflection invariance of the system. In the Pauli-Dirac representation (see Appendix A) the CGNM Hamiltonian is given in full by

$$
\begin{aligned}
& H_{\mathrm{CGNM}}=\sum_{i=1}^{N} \sum_{\mathbf{k}^{\prime}} \frac{1}{\left[\left(\mathbf{k}_{i}^{\prime}\right)^{2}+m^{2}\right]^{1 / 2}}\left\{\left(\mathbf{k}_{i}^{\prime}\right)^{2}\left(b_{\mathbf{k}_{i}^{\prime}, 1}^{\dagger} b_{\mathbf{k}_{i}^{\prime}, 1}-b_{\mathbf{k}_{i}^{\prime}, 2} b_{\mathbf{k}_{i}^{\prime}, 2}^{\dagger}\right)-m\left|\mathbf{k}_{i}^{\prime}\right|\left(b_{\mathbf{k}_{i}^{\prime}, 2} b_{-\mathbf{k}_{i}^{\prime}, 1}+b_{\mathbf{k}_{i}^{\prime}, 1}^{\dagger} b_{-\mathbf{k}_{i}^{\prime}, 2}^{\dagger}\right)\right\} \\
& +\left(\frac{g^{2} m^{2}}{2 L}\right) \sum_{i, j=1}^{N} \sum_{\left(\mathbf{k}^{\prime}, \mathbf{k}^{\prime \prime}, \mathbf{k}^{\prime \prime \prime}, \mathbf{k}^{\prime \prime \prime \prime}\right)}\left\{\frac{1}{\left[\left(\mathbf{k}_{i}^{\prime}\right)^{2}+m^{2}\right]^{1 / 4}\left[\left(\mathbf{k}_{i}^{\prime \prime}\right)^{2}+m^{2}\right]^{1 / 4}\left[\left(\mathbf{k}_{j}^{\prime \prime \prime}\right)^{2}+m^{2}\right]^{1 / 4}\left[\left(\mathbf{k}_{j}^{\prime \prime \prime \prime}\right)^{2}+m^{2}\right]^{1 / 4}}\right\}
\end{aligned}
$$

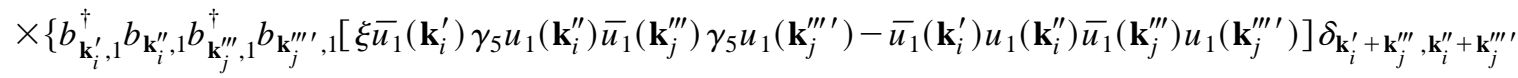

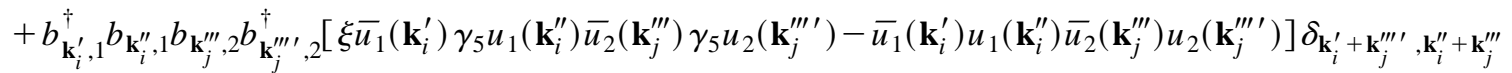

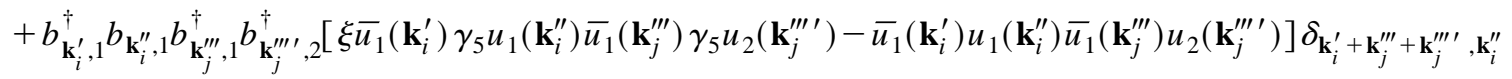

$$
\begin{aligned}
& +b_{\mathbf{k}_{i}^{\prime}, 1}^{\dagger} b_{\mathbf{k}_{i}^{\prime \prime}, 1} b_{\mathbf{k}_{j}^{\prime \prime \prime}, 2} b_{\mathbf{k}_{j}^{\prime \prime \prime}, 1}\left[\xi \bar{u}_{1}\left(\mathbf{k}_{i}^{\prime}\right) \gamma_{5} u_{1}\left(\mathbf{k}_{i}^{\prime \prime}\right) \bar{u}_{2}\left(\mathbf{k}_{j}^{\prime \prime \prime}\right) \gamma_{5} u_{1}\left(\mathbf{k}_{j}^{\prime \prime \prime \prime}\right)-\bar{u}_{1}\left(\mathbf{k}_{i}^{\prime}\right) u_{1}\left(\mathbf{k}_{i}^{\prime \prime}\right) \bar{u}_{2}\left(\mathbf{k}_{j}^{\prime \prime \prime}\right) u_{1}\left(\mathbf{k}_{j}^{\prime \prime \prime \prime}\right)\right] \delta_{\mathbf{k}_{i}^{\prime}, \mathbf{k}_{i}^{\prime \prime}+\mathbf{k}_{j}^{\prime \prime \prime}+\mathbf{k}_{j}^{\prime \prime \prime} \prime}
\end{aligned}
$$




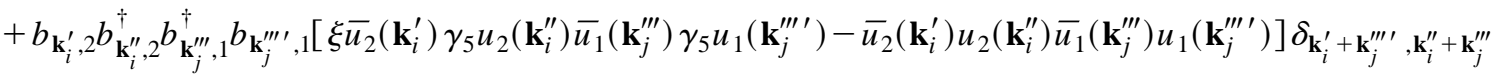

$$
\begin{aligned}
& +b_{\mathbf{k}_{i}^{\prime}, 2} b_{\mathbf{k}_{i}^{\prime \prime}, 2}^{\dagger} b_{\mathbf{k}_{j}^{\prime \prime \prime}, 2} b_{\mathbf{k}_{j}^{\prime \prime \prime \prime}, 2}^{\dagger}\left[\xi \overline{u_{2}}\left(\mathbf{k}_{i}^{\prime}\right) \gamma_{5} u_{2}\left(\mathbf{k}_{i}^{\prime \prime}\right) \bar{u}_{2}\left(\mathbf{k}_{j}^{\prime \prime \prime}\right) \gamma_{5} u_{2}\left(\mathbf{k}_{j}^{\prime \prime \prime \prime}\right)-\bar{u}_{2}\left(\mathbf{k}_{i}^{\prime}\right) u_{2}\left(\mathbf{k}_{i}^{\prime \prime}\right) \overline{u_{2}}\left(\mathbf{k}_{j}^{\prime \prime \prime}\right) u_{2}\left(\mathbf{k}_{j}^{\prime \prime \prime \prime}\right)\right] \delta_{\mathbf{k}_{i}^{\prime}}+\mathbf{k}_{j}^{\prime \prime \prime}, \mathbf{k}_{i}^{\prime \prime \prime}+\mathbf{k}_{j}^{\prime \prime \prime \prime}
\end{aligned}
$$

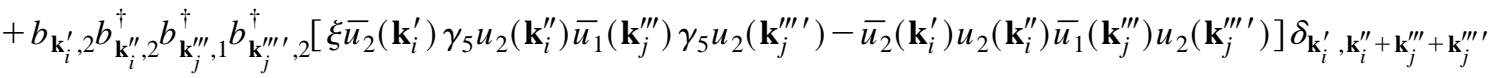

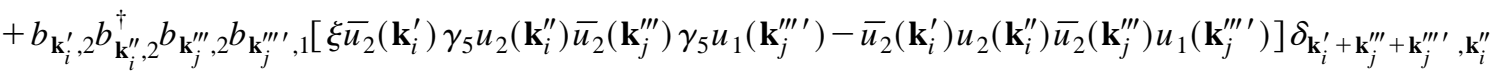

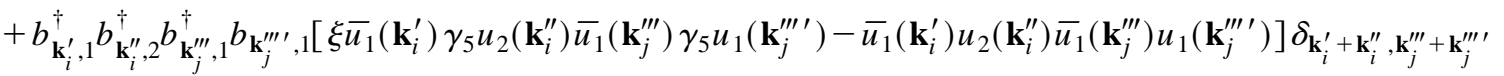

$$
\begin{aligned}
& +b_{\mathbf{k}_{i}^{\prime}, 1}^{\dagger} b_{\mathbf{k}_{i}^{\prime \prime}, 2}^{\dagger} b_{\mathbf{k}_{j}^{\prime \prime \prime}, 2} b_{\mathbf{k}_{j}^{\prime \prime \prime \prime}, 2}^{\dagger}\left[\xi \bar{u}_{1}\left(\mathbf{k}_{i}^{\prime}\right) \gamma_{5} u_{2}\left(\mathbf{k}_{i}^{\prime \prime}\right) \bar{u}_{2}\left(\mathbf{k}_{j}^{\prime \prime \prime}\right) \gamma_{5} u_{2}\left(\mathbf{k}_{j}^{\prime \prime \prime \prime}\right)-\bar{u}_{1}\left(\mathbf{k}_{i}^{\prime}\right) u_{2}\left(\mathbf{k}_{i}^{\prime \prime}\right) \bar{u}_{2}\left(\mathbf{k}_{j}^{\prime \prime \prime}\right) u_{2}\left(\mathbf{k}_{j}^{\prime \prime \prime \prime}\right)\right] \delta_{\mathbf{k}_{i}^{\prime}+\mathbf{k}_{i}^{\prime \prime}+\mathbf{k}_{j}^{\prime \prime \prime \prime}, \mathbf{k}_{j}^{\prime \prime \prime}} \\
& +b_{\mathbf{k}_{i}^{\prime}, 1}^{\dagger} b_{\mathbf{k}_{i}^{\prime \prime}, 2}^{\dagger} b_{\mathbf{k}_{j}^{\prime \prime \prime}, 1}^{\dagger} b_{\mathbf{k}_{j}^{\prime \prime \prime \prime}, 2}^{\dagger}\left[\xi \bar{u}_{1}\left(\mathbf{k}_{i}^{\prime}\right) \gamma_{5} u_{2}\left(\mathbf{k}_{i}^{\prime \prime}\right) \bar{u}_{1}\left(\mathbf{k}_{j}^{\prime \prime \prime}\right) \gamma_{5} u_{2}\left(\mathbf{k}_{j}^{\prime \prime \prime \prime}\right)-\bar{u}_{1}\left(\mathbf{k}_{i}^{\prime}\right) u_{2}\left(\mathbf{k}_{i}^{\prime \prime}\right) \bar{u}_{1}\left(\mathbf{k}_{j}^{\prime \prime \prime}\right) u_{2}\left(\mathbf{k}_{j}^{\prime \prime \prime \prime}\right)\right] \delta_{\mathbf{k}_{i}^{\prime}+\mathbf{k}_{i}^{\prime \prime \prime}+\mathbf{k}_{j}^{\prime \prime \prime}+\mathbf{k}_{j}^{\prime \prime \prime}, 0}
\end{aligned}
$$

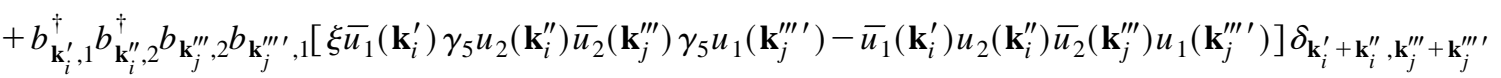

$$
\begin{aligned}
& +b_{\mathbf{k}_{i}^{\prime}, 2} b_{\mathbf{k}_{i}^{\prime \prime}, 1} b_{\mathbf{k}_{j}^{\prime \prime \prime}, 1}^{\dagger} b_{\mathbf{k}_{j}^{\prime \prime \prime}, 1}\left[\xi \overline{u_{2}}\left(\mathbf{k}_{i}^{\prime}\right) \gamma_{5} u_{1}\left(\mathbf{k}_{i}^{\prime \prime}\right) \bar{u}_{1}\left(\mathbf{k}_{j}^{\prime \prime \prime}\right) \gamma_{5} u_{1}\left(\mathbf{k}_{j}^{\prime \prime \prime \prime}\right)-\bar{u}_{2}\left(\mathbf{k}_{i}^{\prime}\right) u_{1}\left(\mathbf{k}_{i}^{\prime \prime}\right) \bar{u}_{1}\left(\mathbf{k}_{j}^{\prime \prime \prime}\right) u_{1}\left(\mathbf{k}_{j}^{\prime \prime \prime \prime}\right)\right] \delta_{\mathbf{k}_{i}^{\prime}+\mathbf{k}_{i}^{\prime \prime}+\mathbf{k}_{j}^{\prime \prime \prime \prime}, \mathbf{k}_{j}^{\prime \prime \prime}} \\
& +b_{\mathbf{k}_{i}^{\prime}, 2} b_{\mathbf{k}_{i}^{\prime \prime}, 1} b_{\mathbf{k}_{j}^{\prime \prime \prime}, 2} b_{\mathbf{k}_{j}^{\prime \prime \prime \prime}, 2}^{\dagger}\left[\xi \overline{u_{2}}\left(\mathbf{k}_{i}^{\prime}\right) \gamma_{5} u_{1}\left(\mathbf{k}_{i}^{\prime \prime}\right) \bar{u}_{2}\left(\mathbf{k}_{j}^{\prime \prime \prime}\right) \gamma_{5} u_{2}\left(\mathbf{k}_{j}^{\prime \prime \prime \prime}\right)-\bar{u}_{2}\left(\mathbf{k}_{i}^{\prime}\right) u_{1}\left(\mathbf{k}_{i}^{\prime \prime}\right) \bar{u}_{2}\left(\mathbf{k}_{j}^{\prime \prime \prime}\right) u_{2}\left(\mathbf{k}_{j}^{\prime \prime \prime \prime}\right)\right] \delta_{\mathbf{k}_{i}^{\prime}+\mathbf{k}_{i}^{\prime \prime}+\mathbf{k}_{j}^{\prime \prime \prime}, \mathbf{k}_{j}^{\prime \prime \prime \prime}} \\
& +b_{\mathbf{k}_{i}^{\prime}, 2} b_{\mathbf{k}_{i}^{\prime \prime}, 1} b_{\mathbf{k}_{j}^{\prime \prime \prime}, 1}^{\dagger} b_{\mathbf{k}_{j}^{\prime \prime \prime}, 2}^{\dagger}\left[\xi \overline{u_{2}}\left(\mathbf{k}_{i}^{\prime}\right) \gamma_{5} u_{1}\left(\mathbf{k}_{i}^{\prime \prime}\right) \bar{u}_{1}\left(\mathbf{k}_{j}^{\prime \prime \prime}\right) \gamma_{5} u_{2}\left(\mathbf{k}_{j}^{\prime \prime \prime \prime}\right)-\bar{u}_{2}\left(\mathbf{k}_{i}^{\prime}\right) u_{1}\left(\mathbf{k}_{i}^{\prime \prime}\right) \bar{u}_{1}\left(\mathbf{k}_{j}^{\prime \prime \prime}\right) u_{2}\left(\mathbf{k}_{j}^{\prime \prime \prime \prime}\right)\right] \delta_{\mathbf{k}_{i}^{\prime}+\mathbf{k}_{i}^{\prime \prime}, \mathbf{k}_{j}^{\prime \prime \prime}+\mathbf{k}_{j}^{\prime \prime \prime \prime}}
\end{aligned}
$$

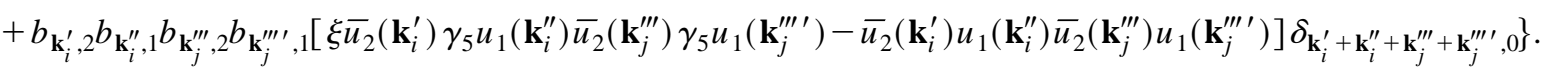

This Hamiltonian is written in a particle basis. In the dynamical equations (35) we have to use the CGNM Hamiltonian in the Nambu quasiparticle basis. The transformed Hamiltonian is obtained in a straightforward way using Eqs. (11) and (30).

\section{APPENDIX C: SELF-CONSISTENT RENORMALIZATION}

Using Eq. (46) we obtain $\left.\sin 2 \varphi_{\mathbf{k}}\right|_{\text {eq }}$ and $\left.\cos 2 \varphi_{\mathbf{k}}\right|_{\mathrm{eq}}$ as

$$
\begin{gathered}
\left.\sin 2 \varphi_{\mathbf{k}}\right|_{\text {eq }}=\frac{-(-1)^{n} m|\mathbf{k}|[1-(b+d)]}{k_{0}\left[k^{2}+m^{2}(b+d)^{2}\right]^{1 / 2}}, \\
\left.\cos 2 \varphi_{\mathbf{k}}\right|_{\text {eq }}=\frac{\left[k^{2}+m^{2}(b+d)\right]}{k_{0}\left[k^{2}+m^{2}(b+d)^{2}\right]^{1 / 2}} .
\end{gathered}
$$

Substituting Eq. (C1) in $I_{1}$ and $I_{2}$ given in Eq. (37), taking $\nu_{\mathbf{k}, 1}=\nu_{\mathbf{k}, 2}=0$, and performing the integration, we have

$$
\begin{gathered}
I_{1}=\int_{-\Lambda}^{+\Lambda} \frac{d k}{\left(k^{2}+m^{2}\right)} \frac{\left[\mathbf{k}^{2}+m^{2}(b+d)\right]}{\left[k^{2}+m^{2}(b+d)^{2}\right]}=a+b \ln \left(\frac{\Lambda^{2}}{m^{2}}\right), \\
a=\frac{2 d}{\left[(1+d)^{2}-1\right]^{1 / 2}} \arctan \left[(1+d)^{2}-1\right]^{1 / 2}+\ln \left[\frac{4}{(1+d)^{2}}\right] \quad \text { and } \quad b=1 \quad \text { when }(1+d)^{2}>1, \\
a=\frac{d}{\left[1-(1+d)^{2}\right]^{1 / 2}} \ln \left[\frac{1+\left\{1-(1+d)^{2}\right\}^{1 / 2}}{1-\left\{1-(1+d)^{2}\right\}^{1 / 2}}\right]+\ln \left[\frac{4}{(1+d)^{2}}\right] \quad \text { and } \quad b=1 \quad \text { when }(1+d)^{2}<1, \\
I_{2}=(b+d-1) \int_{-\Lambda}^{\Lambda} \frac{d k}{\left(k^{2}+m^{2}\right)} \frac{k^{2}}{\left[k^{2}+m^{2}(b+d)^{2}\right]^{1 / 2}}=c+d \ln \left(\frac{\Lambda^{2}}{m^{2}}\right) .
\end{gathered}
$$

This shows that $d$ is not determined by self-consistency. For $b=1$, 


$$
\begin{gathered}
c=\frac{-2 d}{\left[(1+d)^{2}-1\right]^{1 / 2}} \arctan \left[(1+d)^{2}-1\right]^{1 / 2}+d \ln \left[\frac{4}{(1+d)^{2}}\right] \quad \text { when }(1+d)^{2}>1, \\
c=\frac{-d}{\left[1-(1+d)^{2}\right]^{1 / 2}} \ln \left[\frac{1+\left\{1-(1+d)^{2}\right\}^{1 / 2}}{1-\left\{1-(1+d)^{2}\right\}^{1 / 2}}\right]+d \ln \left[\frac{4}{(1+d)^{2}}\right] \quad \text { when }(1+d)^{2}<1 .
\end{gathered}
$$

This shows that the Ansätze (44) and (45) are self-consistent. Equations (C2) and (C3) give the values of the constants $a, b$, and $c$.

[1] M. Samiullah, O. Éboli, and S.-Y. Pi, Phys. Rev. D 44, 2335 (1991), and references therein; D. Boyanovski, H. J. de Vega, and R. Holman, ibid. 49, 2769 (1994); F. L. Braghin, C. Martin, and D. Vautherin, Phys. Lett. B 348, 343 (1995).

[2] M. Ploszajczak and M. Rhodes-Brown, Phys. Rev. D 33, 3686 (1986); H.-Th. Else, M. Gyulassy, and D. Vasak, Phys. Lett. B 177, 402 (1986); C. Miug Ko, Qi Li, and Renchuan Wang, Phys. Rev. Lett. 59, 1084 (1987).

[3] M. C. Nemes and A. F. R. de Toledo Piza, Physica A 137, 367 (1986), and references therein.

[4] O. Éboli, S.-Y. Pi, and R. Jackiw, Phys. Rev. D 37, 3557 (1988); A. Kovner and B. Rosenstein, Ann. Phys. (N.Y.) 187, 449 (1988); R. Jackiw, Physica A 158, 269 (1989).

[5] A. K. Kerman and D. Vautherin, Ann. Phys. (N.Y.) 192, 408 (1989); A. K. Kerman and C. Y. Lin, ibid. 241, 185 (1995).

[6] J. des Cloiseaux, in The Many-Body Physics, edited by C. de Witt and R. Balian (Gordon and Breach, New York, 1968).

[7] Shan-Jin Chang, Phys. Rev. D 12, 1071 (1975).

[8] A. F. R. de Toledo Piza, in Time-Dependent Hartree-Fock and Beyond, edited by K. Goeke and P.-G. Reinhardt, Lectures Notes in Physics Vol. 171 (Springer-Verlag, Berlin, 1982); M. C. Nemes and A. F. R. de Toledo Piza, Phys. Rev. C 27, 862 (1983); B. V. Carlson, M. C. Nemes, and A. F. R. de Toledo Piza, Nucl. Phys. A457, 261 (1986).
[9] P. Buck, H. Feldmeier, and M. C. Nemes, Ann. Phys. (N.Y.) 185, 170 (1988).

[10] L. C. Yong and A. F. R. de Toledo Piza, Phys. Rev. D 46, 742 (1992); L. C. Yong, Doctoral thesis, University of São Paulo, 1991.

[11] D. J. Gross and A. Neveu, Phys. Rev. D 10, 3235 (1974).

[12] P. L. Natti, Doctoral thesis, University of São Paulo, 1995.

[13] A. Kerman and T. Troudet, Ann. Phys. (N.Y.) 154, 456 (1984).

[14] R. Daschen, B. Hasslacher, and A. Neveu, Phys. Rev. D 12, 2443 (1975).

[15] Y. Nambu and G. Jona-Lasinio, Phys. Rev. 122, 345 (1961).

[16] E. Witten, Nucl. Phys. B145, 110 (1978).

[17] B. Berg, M. Karowski, V. Kurac, and P. Weisz, Nucl. Phys. B134, 125 (1978).

[18] P. R. I. Tommasini, Doctoral thesis, University of São Paulo, 1995.

[19] B. Rosenstein, and A. Kovner, Phys. Rev. D 40, 523 (1989); R. Pausch, M. Thies, and V. L. Dolman, Z. Phys. A 338, 441 (1991).

[20] A. F. R. de Toledo Piza, in Proceedings of the IX Workshop in Nuclear Physics, Buenos Aires, Argentina, 1986, edited by A. O. Machiavelli, H. M. Sofia, and E. Ventura (World Scientific, Singapore, 1987). 